\title{
1 ExPRSweb - An Online Repository with Polygenic Risk Scores for
}

\section{Common Health-related Exposures}

4 Ying $\mathrm{Ma}^{1,2}$, Snehal Patil ${ }^{1,2}$, Xiang Zhou ${ }^{1,2,3}$, Bhramar Mukherjee ${ }^{1,2,3,4,5,6,7}$ and Lars G.

5 Fritsche $e^{1,2,3,5,7, *}$

6

$7{ }^{1}$ Department of Biostatistics, University of Michigan School of Public Health, Ann Arbor, Michigan

8 48109, United States of America

$9{ }^{2}$ Center for Statistical Genetics, University of Michigan School of Public Health, Ann Arbor, Michigan

10 48109, United States of America

$11{ }^{3}$ Center for Precision Health Data Science, University of Michigan School of Public Health, Ann

12 Arbor, Michigan 48109, United States of America

$13{ }^{4}$ Department of Epidemiology, University of Michigan School of Public Health, Ann Arbor, Michigan

14 48109, United States of America

155 University of Michigan Rogel Cancer Center, University of Michigan, Ann Arbor, Michigan 48109,

16 United States of America

$17{ }^{6}$ Michigan Institute for Data Science, University of Michigan, Ann Arbor, Michigan 48109, United

18 States of America

197 These authors jointly supervised this work

20 * Correspondence should be addressed to L.G.F. (larsf@umich.edu) 


\section{Abstract}

23 Complex traits are influenced by genetic risk factors, lifestyle, and environmental

24 variables, so called exposures. Some exposures, e.g., smoking or lipid levels, have

25 common genetic modifiers identified in genome-wide association studies. Since

26 measurements are often unfeasible, Exposure Polygenic Risk Scores (ExPRSs) offer

27 an alternative to study the influence of exposures on various phenotypes. Here, we

28 collected publicly available summary statistics for 28 exposures and applied four

29 common PRS methods to generate ExPRSs in two large biobanks, the Michigan

30 Genomics Initiative and the UK Biobank. We established ExPRS for 27 exposures and

31 demonstrated their applicability in phenome-wide association studies and as predictors

32 for common chronic conditions. Especially, the addition of multiple ExPRSs showed, for

33 several chronic conditions, an improvement compared prediction models that only

34 included traditional, disease-focused PRSs. To facilitate follow-up studies, we share all

35 ExPRS constructs and generated results via an online repository called ExPRSweb. 


\section{Introduction}

A central challenge in genetics is to understand inherited factors underlying

39 complex traits and disorders. Substantial efforts in the past decade, especially genome-

40 wide association studies (GWAS), have successfully uncovered genetic variants

41 associated with a plethora of traits ${ }^{1}$. However, translating these to disease etiology or to

42 predict outcomes is not straightforward. Most genetic risk variants have weak and

43 sparse marginal effects, accounting for only a small fraction of the phenotypic variation,

44 even for highly heritable traits ${ }^{2-4}$. Consequently, incorporating information across genetic

45 variants is necessary for assessing the predisposition of complex traits.

$46 \quad$ The construction of polygenic risk score (PRS) is among the widely used

47 approaches to translate genetic information into a disease risk ${ }^{5,6}$. A PRS is formed as a

48 summation of an individual's risk alleles, weighted by the effect sizes obtained from an

49 external GWAS. PRS methods rely on the polygenicity of complex traits and vary in

50 data input, model assumptions, validation procedures, and whether functional

51 annotations or pleiotropic information is incorporated ${ }^{7}$.

52 In addition to genetic risk factors, lifestyle, and environmental variables, so called

53 exposures, can impact disease risks. For example, high body mass index, smoking,

54 blood lipid levels, and pre-existing type 2 diabetes (T2D) were recognized as prominent

55 risk factors for cardiovascular disease ${ }^{8}$, respiratory diseases ${ }^{9}$, and cancers ${ }^{10,11}$. Given

56 the relevance for these often modifiable risk factors for morbidity and mortality,

57 exposure information is pivotal for precision prevention ${ }^{10}$. However, even common

58 exposures are not always available, especially when using electronic health records

59 (EHRs). Furthermore, data can be prone to measurement error, bias, and non-random 
60 missingness ${ }^{12,13}$. Yet, some exposures have a heritable component identifiable through

61 GWAS ${ }^{14,15}$ and thus offer the opportunity to construct Exposure PRSs (ExPRSs).

As genetic proxies at the individual level, ExPRSs have been used in many

63 applications, e.g., risk prediction and stratification ${ }^{16-18}$, predicting exposures ${ }^{19}$,

64 instruments for Mendelian randomization analyses, or phenome-wide association

65 studies (PheWASs) ${ }^{20-23}$. Including ExPRSs to prediction models could improve disease

66 diagnosis, screening, therapeutic interventions, and precision medicine approaches.

67 PheWAS with ExPRSs may identify clinical phenotypes associated with a modifiable

68 exposure and thereby highlight diseases whose onset might be influenced by early

69 intervention or behavioral / lifestyle modification ${ }^{20}$. Noteworthy, ExPRSs capture the

70 genetic predisposition of an exposure assigned at birth, but not the environmental

71 influence, thus leaving a large proportion of the exposure's variance unexplained. Still,

72 the identification of associations between diseases and ExPRSs may help to tease

73 apart the interplay of genetic and environmental pathways through which they influence

74 disease risk.

75 The emerging utility of PRSs is evidenced via the accumulation of more than

76 1,000 PRS-related articles indexed in PubMed since $2009{ }^{24}$ and spurred by significant

77 advances in PRS methods ${ }^{7}$. Despite the rise in popularity, their transition into clinical

78 settings is often limited by lack of transparency, compatibility, and reproducibility across

79 cohorts. Therefore, a ExPRS resource that integrates adequate information for

80 constructing, evaluating, and utilizing ExPRSs to accelerate ExPRS-related research is

81 desirable and necessary. Recently, we established "Cancer PRSweb", an interactive,

82 online repository with cancer PRSs for 35 common cancer traits ${ }^{20}$. Building upon our 
83 previous work, we present ExPRSweb, a uniform analytic framework and an extension

84 of PRSweb that specifically focusses on ExPRSs for 28 common exposures.

85 By using available exposure GWAS summary statistics and two large biobanks,

86 the Michigan Genomics Initiative (MGI) and the UK Biobank (UKB), we generated

87 ExPRS with four methods varying in complexity and modeling (i.e., linkage

88 disequilibrium clumping and $p$-value thresholding $[\mathrm{C}+\mathrm{T}]$, Lassosum, Deterministic

89 Bayesian Sparse Linear Mixed Model [DBSLMM], and PRS-CS, a Bayesian method

90 with continuous shrinkage priors) $)^{25-30}$. We also highlight ExPRSs applications including

91 PheWAS, risk stratification and prediction of common chronic conditions. For the latter,

92 we evaluated the predictive performance of single and multiple ExPRSs when combined

93 with disease specific PRS and could show substantial improvement for several trait. We

94 also contrasted these predictors with "Poly-Exposure Risk Scores" (PXSs) which

95 integrate multiple measured exposures.

96 In absence of high-quality exposure data on many individuals ExPRSs can serve

97 as surrogates if one has genotype data on a larger and more representative sample.

98 Our repository ExPRSweb unlocks access to over 300 ExPRSs for 27 different

99 exposures and facilitates scientific collaboration to strengthen their future application. 


\section{Results}

\section{Descriptive Characteristics of Study Cohorts}

102 For the generation and analysis of ExPRSs, we used two analytical datasets that were

103 restricted to unrelated participants of broad European ancestry encompassing 46,782

104 individuals in MGI and 408,595 individuals in UKB (Table 1; Subjects and Methods) ${ }^{31-}$

$105{ }^{33}$. The different prevalence of binary exposures and common chronic conditions in MGI

106 and UKB likely reflect the characteristics of a hospital-based study (MGI) and a

107 healthier, population-based study (UKB), respectively (Table 1, Table S1). For

108 example, there are marked differences between MGI and UKB regarding hypertension $109(49.8 \%$ vs. $27.0 \%)$, diabetes ( $21.4 \%$ vs. $7.2 \%)$, and lung cancer (2.2\% vs. $1.0 \%)$. Also,

110 overweight individuals ( $74.7 \%$ vs. $66.8 \%)$ and smokers ( $49.2 \%$ vs. $39.4 \%)$ were more

111 frequent in MGI (Figure S1).

\section{Heritability Estimates}

114 In total, we identified 82 sets of GWAS summary statistics for 28 different exposures (21

115 quantitative, 7 binary) that had matching exposure data in MGI and/or UKB; 52 solely

116 based on UKB data and 30 on large GWAS (Table 2, Table S2). For each set, we

117 estimated the narrow sense heritability ${ }^{34}$ as PRSs are closely connected to it and

118 because one PRS method (DBSLMM) relies on these estimates. After excluding three

119 GWAS sets with negative $\mathrm{h}^{2}$ estimates, we observed heritability estimates between

1200.003 (sleep apnea) and 0.518 (height) that were in line with previous studies (Table

121 S3) ${ }^{4,35-39}$. Still, estimates from GWAS on the same exposure often varied (e.g., 
$122 h^{2}$ [height]: $0.012-0.518$ or $h^{2}[$ vitamin $D]: 0.009-0.100$ ) implying different underlying

123 frameworks (Figure S2).

\section{ExPRS Evaluation}

126 Following the scheme in Figure 1, we generated 514 ExPRSs (379 for 25 exposures in

$127 \mathrm{MGI}$ and 135 for 17 exposures in UKB; Table S4) and assessed association, overall

128 performance, accuracy, and discrimination. A total of 336 ExPRSs for 27 exposures

129 were nominally significant and positively associated with their corresponding exposures

130 in MGI (262 ExPRSs; 24 exposures) and in UKB (74 ExPRSs; 14 exposures) and

131 analyzed further (Table S4).

132

133 Performance comparison across methods

134 For the method comparison we focus on MGI having a more comprehensive set of

135 exposures covered by ExPRSs. PRS-CS produced the best performing ExPRSs for 18

136 of the 24 exposures consistent with previous benchmarking (Table 3, Figure 2, and

137 Figure S3) $)^{40-42}$. Lassosum excelled for the alcohol and smoker exposure, DBSLMM for

138 lipid levels, and both $\mathrm{C}+\mathrm{T}$ implementations for exposures with low heritability, e.g.,

139 vitamin B12 and D (Figure 2). Further, we found that the $\mathrm{C}+\mathrm{T}$ implementation that uses

140 dosages for LD clumping had a slight edge over the one using best guess genotypes,

141 confirming previous findings ${ }^{43}$. Overall, these results suggested the methods'

142 performances differed by trait showcasing the benefit of screening multiple methods.

144 Performance across exposures 
145 Again, focusing on MGl, we selected for each exposure the ExPRS with the lowest

146 association p-value among its method / exposure GWAS combinations (Table S4). For

147 quantitative exposures the Pearson's correlation $r$ with their corresponding ExPRS

148 ranged from 0.049 (Vitamin B12) to 0.373 (Height). For binary exposures, the area

149 under the covariate-adjusted area under the ROC curve (AAUC) ranged from 0.524

150 (insomnia) to 0.637 (T2D), confirming only modest discrimination by PRS for complex

151 traits ${ }^{44}$. The ExPRSs' performance generally agreed with the ranking of their heritability

152 estimates (Figure S4 and S5).

153

154 Performance comparison across cohorts

155 Like MGI, we selected in UKB for each of the 14 exposures the ExPRS that reached the 156 strongest association (Table S5): 6 were based on Lassosum, 4 on PRS-CS, 3 on C+T

157 (DS), and 1 on $\mathrm{C}+\mathrm{T}(\mathrm{GT})$. In contrast to MGI, Lassosum outperformed the other

158 methods in UKB (Figure S6 and S7). The inconsistencies across cohorts might be the

159 result of different underlying GWAS sets, i.e., for UKB ExPRSs we only relied on non-

160 UKB studies to avoid overfitting. Also, the methods' varying tuning procedures

161 especially for Lassosum and $\mathrm{C}+\mathrm{T}$ might be affected by the larger sample sizes in UKB.

162 For ExPRSs of quantitative exposures, the correlation with their corresponding

163 exposures ranged from 0.015 (alcohol consumption) to 0.326 (height) (Table S5). For

164 binary exposures, the AAUC ranged from 0.505 (hypertension) to 0.825 (T2D). When

165 comparing ExPRSs on exposures that were present in both cohorts, we found generally

166 consistent performances for quantitative traits like C-reactive protein, creatine, vitamin

167 D, and height, while for some binary traits such as the T2D (AAUC MGI: $_{0.64,}$ AAUCukB: 
168 0.83) and smoking (AAUCMGI: 0.61, AAUCukB: 0.77), AAUC differed substantially (Table

169 S6). Noteworthy, the estimates in UKB might be heightened due to undetected,

170 overlapping samples between their discovery GWAS and the UKB cohort ${ }^{45,46}$, or caused

171 by to the cohort's larger effective sample sizes.

\section{Correlations of ExPRSs across exposures}

174 Next, we assessed the relationships between ExPRSs and exposures in MGI. Figure 3

175 displays the pairwise correlation between 15 quantitative exposures, between their 15

176 corresponding ExPRSs, and between the ExPRSs and the quantitative exposures in

177 MGI. The correlations between the quantitative exposures indicated positive and

178 negative relationships ( $r$ between -0.1 and 0.92 ; Figure $3 \mathrm{~A}$ ), the strongest between

179 closely related exposures: $r[T C, L D L]=0.92, r[e G F R$, creatine $]=-0.84$, and r[SBP,

$180 \mathrm{DBP}]=0.53$. The former two can be attributed to their underlying equations and related

181 measurements, while the linear relationship between SBP and DBP is well-

182 established ${ }^{47-49}$. Several of the other observed correlations are also well-documented

183 often reflecting related disease etiologies ${ }^{50-52}$. Similar but more attenuated patterns

184 were seen for the ExPRSs whose correlations ranged from -0.78 to 0.72 (Figure 3B).

185 The often lower pairwise correlations (e.g., r[PRSTc, PRSLDL] $=0.72$ and r[PRS eGFR,

186 PRS $_{\text {creatine }}=-0.78$ ) were expected because ExPRSs capture only a fraction of the

187 exposure's variance (see diagonal of Figure $3 \mathrm{C}$ ). The consistent patterns suggested

188 that several ExPRSs can replicate correlations of measured exposures relatively well

189 and thus might be suitable surrogates for exposures, especially for studies where

190 measurements might not be feasible or likely be biased $50,53,54$. 


\section{ExPRS Applications}

193 Phenome-wide Association Analyses

194 One application of ExPRSs is their use as predictors for phenome-wide association 195 studies (PheWASs) to uncover phenotypes with a shared genetic component and thus

196 disorders that might benefit from an early intervention. We showcase such ExPRS

197 PheWASs by analyzing all 24 selected ExPRSs across up to 1685 EHR-derived

198 phenotypes (PheCodes) in MGI (Table S7). In total, we observed phenome-wide

199 significant associations between 22 ExPRSs and 440 phenotypes (Bonferroni-corrected

200 threshold at $\mathrm{P}<0.05$ / 1685; Table S8). Overall, the number and the strength of

201 observed associations seem to depend on the exposures' impact and heritability. For

202 example, the PheWAS with the BMI ExPRS uncovered 329 associated phenotypes

203 while the Vitamin B-12 ExPRS PheWAS only revealed two associations with closely

204 related phenotypes. Besides the expected associations between BMI PRS and obesity-

205 related phenotypes ( $1.66<$ OR $<2.14$, e.g., obesity, morbid obesity, and overweight),

206 we also observed significant phenome-wide associations with hypertension (OR: 1.33

207 [1.30,1.36]), T2D (OR: $1.41[1.37,1.45])$, osteoarthrosis (OR: 1.15 [1.12,1.17]), and

208 sleep apnea (OR: 1.28 [1.25,1.31]); all previously reported for BMI55-58 (Figure 4A;

209 Table S8). The PheWAS with measured BMI revealed consistent associations (Figure

210 4C; Table S9) though with larger effects: hypertension (OR: 1.88 [1.84, 1.93]), type 2

211 diabetes (OR: $2.00[1.95,2.06])$, osteoarthrosis (OR: 1.29 [1.27,1.32]), and sleep apnea

212 (OR: $2.24[2.18,2.30])$. 
To assess whether these associations were driven by exposed individuals, i.e.,

214 individuals affected by a binary exposure or by low or high exposure values, we also

215 performed "Exclusion-PRS-PheWAS" analyses where we excluded such exposed

216 individuals to remove direct and indirect associations of the exposure and potential

217 treatment effects (see Methods). While this exclusion of individuals markedly

218 decreased sample sizes and thus power, we identified 198 phenotypes that remained

219 significantly associated with 17 ExPRSs in the Exclusion PRS PheWAS $(\mathrm{P}<0.05$ /

220 1685; Table S8). For example, in the Exclusion PheWAS with the BMI ExPRS the

221 associations with hypertension (OR: $1.17[1.12,1.23])$ and T2D (OR: $1.18[1.09,1.27])$

222 remained statistically significant (Figure 4B, Table S8). However, while the analysis of

223 individuals with healthy BMI removed most of the obesity or overweight phenotypes, a

224 strong association remained between BMI ExPRS and bariatric surgery (OR: 2.66 [2.08,

$2253.41])$. A closer inspection revealed that 73 of 1509 MGl participants who underwent

226 bariatric surgery had recorded median BMI values that fell in the healthy BMI range

$227(18.5<\mathrm{BMI}<25)$ indicating the BMI ExPRS's ability to capture pre-treatment

228 exposures. Most interestingly, the corresponding Exclusion PheWAS with measured

$229 \mathrm{BMI}$ as predictor revealed many association signals that were reversed compared to the

230 Exclusion-PRS-PheWAS (Figure 4C and 4D, Table S8 and S9). This finding might

231 reflect biased measurements, e.g., due to treatment or interventions that result in

232 normal BMI values, or the measured BMI's inability to capture central obesity ${ }^{59}$.

233 We performed similar sets of PheWASs in UKB. While based on a separate

234 ExPRS generations restricted to UKB-independent GWAS summary statistics, most of

235 the strong associations seen in the MGl were also seen in the UKB ExPRS PheWASs, 
236 e.g., obesity associated with T2D PRS (ORMGl: $1.71[1.15,1.20]$ and ORukB: 1.63 [1.60,

237 1.66]; Figure S8 and S9). Due to the larger sample sizes in the UKB compared to MGI

238 (Table 2), we often observed more and stronger secondary trait associations (Table

$239 \mathrm{~S} 10$ and S11).

240 In general, we found that agnostic ExPRS PheWASs can provide valuable insights into

241 exposure - phenotype relationships, many of which were previously reported for

242 measured exposures. However, thorough investigations are needed to distinguish

243 between spurious and genuine signals.

244

245 Improving Prediction Models for Common Chronic Conditions

246 As many exposures are important risk factors for common chronic

247 conditions $^{9,60,61}$, we performed analyses with a specific emphasis on 27 chronic

248 conditions whose algorithms are used for Medicaid and Medicare claims and available

249 from the Chronic Condition Data Warehouse (CCW, Table S12) ${ }^{62}$. Since these were

250 developed for the US health system and lack transferability to the UK, we limited our

251 analysis to MGI. Related chronic conditions were already covered in the phenome-wide

252 PheCode-based association analyses, therefore this targeted analysis of "real world"

253 phenotype algorithms aims to evaluate the ExPRSs' abilities to improve predictions.

254 Basically, we are interested to see if a prediction model that solely relies on a GWAS-

255 based PRS for a chronic condition "Y" (YPRS) can be augmented with additional

256 ExPRSs.

257 As a first step, we explored the association between the 27 conditions and the 24

258 ExPRSs. We found that even after excluding the directly related condition/exposure 
259 pairs (e.g., hypertension/SBP ExPRS, Hyperlipidemia/TC ExPRS, etc.) all included 24

260 ExPRSs showed a nominally significant association with at least one condition at $\mathrm{P}<$

2610.05 (Table S13). Vice versa, 26 of the 27 conditions were nominally significant

262 associated with at least one ExPRS substantiating the exposures' relevance. However,

263 none of the ExPRSs was associated with Alzheimer's Disease though many of the

264 included exposures were reported risk factors ${ }^{63}$. The strongest risk increasing effect

265 was seen for BMI ExPRS and diabetes (OR: 1.393 [1.357, 1.430]), while the strongest

266 protective effect was seen for HDL ExPRS and diabetes (OR: $0.823[0.803,0.844])$

267 (Table S13).

268 Considering the relatively poor predictive performance of single ExPRSs for

269 chronic conditions and that some of the chronic conditions were associated with several

270 ExPRSs (Table S13), we next assessed whether the combination of ExPRS

271 ("multiExPRS", see Methods) can improve risk prediction of models that only include

272 YPRSs (Table S14).

273 Due to the required cross-validation, limited sample sizes, and limited availability

274 of YPRS (Table S15), we restricted our comparisons to 12 conditions (Table S12 and

275 S15). We found that adding multiple ExPRSs enhanced models for several conditions

276 (e.g., stroke/transient ischemic attack, heart failure, lung cancer, hypertension, chronic

277 kidney disease, asthma; Table S16, Figure 5). For example, the AAUC for predicting

278 hypertension increased from 0.627 to 0.637 when adding multiple ExPRSs (BMI, C

279 reactive protein, drinking status, fast plasma glucose, HDL, height, smoking status,

280 T2D, triglycerides, apnea, and insomnia). In contrast, the addition of ExPRSs did not

281 improve prediction accuracy for other conditions (e.g., glaucoma, prostate cancer, 
282 colorectal cancer, and atrial fibrillation). Nevertheless, the ability of specific ExPRSs to 283 improve predictions indicate that some of the YPRS often do not capture the entirety of

284 an individual's genetic predisposition, likely reflecting the lack of power of the condition's

285 discovery GWAS compared to exposure GWASs which due to larger sample sizes and 286 continuous measurements are often better powered.

287 Since these predictions yielded only moderate to poor discrimination (AAUC $<0.66$ ), we 288 also evaluated the ExPRSs' ability to augment risk stratification with YPRS, i.e., to 289 define subsets of individuals at high risk for the 12 conditions (Figure S10, Table S18 290 and S17). All YPRS were by themselves able to significantly enrich cases in their

291 distributions' tails, though not across all five top percentiles (1, 5, 10, and $25 \%)$. For 292 example, only 8 YPRSs could significantly enrich cases in the top $1 \%$ at $\mathrm{P}<0.05$ with 293 OR ranging from $1.79(95 \% \mathrm{Cl}: 1.37,2.35$; asthma) to $7.13(95 \% \mathrm{Cl}: 5.57,9.13$; atrial 294 fibrillation).

295 Adding the combined ExPRSs (multiExPRSs) to the "YPRS only model"

296 improved the enrichment of cases for 10 of the 12 conditions when considering the top $2971 \%$. A major improvement was seen for the enrichment of cases with heart failure 298 (YPRS: OR: 1.13 [0.74, 1.72] vs YPRS + multiExPRS: OR: $2.72[1.94,3.81])$ and with 299 hypertension (YPRS: OR: 2.96 [2.31,3.80] YPRS + multiExPRS: OR: 3.73 [2.94,4.73]). 300 However, adding multiple ExPRS negatively affected the enrichment of cases with atrial 301 fibrillation (YPRS: OR: 7.13 [5.57, 9.13] vs YPRS + multiExPRS: OR: $6.21[4.82,8.00])$.

302 Similar but less pronounced enrichments of cases were seen for the top $2 \%$, top $5 \%$, 303 top 10\%, and top 25\% (Figure S10, Table S18). 
Our explorations confirmed that individuals in the tails of PRS distributions are

305 most informative for risks of chronic conditions ${ }^{64}$. Further, the consistent gain in risk

306 stratification by adding multiple ExPRS highlights their potential use.

309 multiExPRSs) with poly-exposure scores (PXSs) that are based on measured /

310 collected exposure data as previously described for type 2 diabetes ${ }^{65}$. Again, focusing

311 on the 12 conditions (Table S12), we created a PXS for each condition in the MGI

312 cohort using up to 24 of 27 available exposures (Subjects and Methods). The number

313 of incorporated exposures ranged from 7 (glaucoma) to 19 (chronic kidney disease)

314 (Table S19 and S20). Though the evaluation cohorts were different in size, we

315 observed that the PXSs mostly showed better discrimination than the models that only

316 relied on PRSs (YPRS, multiExPRS, or YPRS + multiExPRS), except for colorectal,

317 prostate, and female breast cancer which underperformed (Figure S11, Table S18).

318 Since PXSs were only obtainable for people who had complete data for each included

319 exposure, they were only available for a small fraction of the genotyped MGI individuals

320 for which YPRSs and ExPRSs were obtainable (2.5 - 18.4\%; Glaucoma: 56.0\%).

321 Furthermore, the proportion of genotyped individuals with complete exposure data for

322 their PXSs was significantly different between cases and controls for 9 of the 12

323 analyzed conditions, indicating non-random missingness of exposures in the MGI EHR

324 that likely biased the analysis. The most extreme example was chronic kidney disease

325 with cases being about four times more likely than controls to have complete exposure

326 data for their PXS (OR $3.9[3.5,4.4], \mathrm{P}=31 . \times 10^{-107}$; Table S21). 


\section{Online Visual Catalog: ExPRSweb}

328 In our current study, we generated and evaluated hundreds of ExPRSs in which

329 predictive properties differed between GWAS source, exposure, method, and/or

330 evaluation cohort (Table S4). To enable an exploration of the ExPRS for 27 different

331 exposures, we created a new PRSweb 20 instance called ExPRSweb (see Web

332 Resources) that includes detailed metrics (association, performance, discrimination,

333 and accuracy) and allows the selection of ExPRSs based on properties for specific

334 applications. The tables, like Table 3 and Table S5, can be sorted, filtered, or

335 downloaded. ExPRSweb also offers detailed information about each ExPRS, including

336 GWAS source(s), LD reference panels and the included risk variants, effect/non-effect

337 alleles, and weights. ExPRSweb also links to interactive ExPRS-PheWAS results for

338 their evaluation cohort, 


\section{Discussion}

340 In this study, we have constructed and evaluated a large set of ExPRSs using 79 sets of

341 GWAS summary statistics, applied various PRS methods and while doing so, created

342 over 514 ExPRSs of which 336 showed promising performance for 27 different

343 exposures in MGI and/or UKB.

We explored the performance of ExPRSs across methods, GWAS sources, and

345 two cohorts and observed two key points that might be helpful to strategize future

346 ExPRS generation projects: First, large exposure GWAS with higher SNP heritability

347 estimates usually also resulted in the most predictive ExPRS. Second, there was no

348 "one size fits all" recipe for ExPRSs, i.e., we recommend the screening of multiple

349 methods.

350 While there is a wide range of health-related exposures ${ }^{66-68}$, we focused on 28

351 exposures for which we could find GWAS from external full summary statistics and

352 which sufficiently measured samples in MGI and/or UKB. The exposures can roughly be

353 categorized into cardiovascular, renal biomarkers, vitamin levels, blood sugar levels,

354 women's health, anthropometric measurements, vitals, health behaviors, and

355 preexisting conditions. However, other relevant exposure were not explored in this

356 study, e.g., dietary exposures (e.g., milk consumption, coffee consumption ${ }^{69,70}$ ),

357 telomere length ${ }^{71}$, and other biomarkers (e.g., Transforming growth factor beta (TGF-

358 beta $)^{72,73}$, circulating microRNA miR-34b (Ref. ${ }^{74}$ ). While some exposures also have

359 GWAS summary statistics available (e.g., coffee consumption, milk consumption

360 summary statistics from UKB GWAS efforts), the exposures were not measured in MGI

361 and thus could not be evaluated at this time. 
363 PRS-CS), all of which are computationally efficient, but skipped other new methods that

364 have been proposed (SBayesR, LDPred, NPS, SCT) ${ }^{75-78}$; but often require massive

365 computational resources, especially for large cohorts like UKB and MGl. Additionally,

366 several alternative methods were reported to improve predictive power by incorporating

367 external information (e.g., functional annotations, pleiotropy across multiple traits), e.g.,

368 LDpred-funct $^{79}$, AnnoPred ${ }^{80}$ and MTGBLUP ${ }^{81}$. Future implementations and systematic

369 evaluations of these alternative choices are needed to further the availability of well-

370 powered ExPRSs and their applications.

We evaluated ExPRSs in cohorts of broadly European ancestry because of the

372 limited diversity in MGI and UKB. However, the lacking transferability across ancestry

373 groups increases the need to also construct ExPRS for non-European ancestry

374 groups ${ }^{29,77,82-84}$. While efforts are underway to develop cross-ancestry PRS methods to

375 increase transferability, ultimately an increased diversity in datasets is needed to

376 counteract the European ancestry bias in GWAS that is passed on to PRS research ${ }^{85,86}$.

377 Our explorations of ExPRSs, mainly in the MGI cohort, revealed that some of the

378 ExPRSs could be good surrogates for exposures and enable meaningful association

379 analyses across medical phenomes or a collection of chronic conditions. Also, the

380 combination of ExPRSs can improve predictions and risk stratification beyond the

381 YPRSs, e.g., for asthma, heart failure, or hypertension. Yet, for some of the studied

382 conditions, the additional of multiple ExPRSs did not improve models that already

383 included YPRSs. This suggests that YPRSs, if based on very large sample sizes, might 
384 already have captured most of the genetic risk profiles reflecting direct and indirect

385 (exposure mediated) risk effects.

There are other applications of ExPRSs that we did not explore but that gained

387 attention in the recent years, e.g., mediations analyses to study polygenic pleiotropy ${ }^{87}$

388 or their use as instrumental variables in Mendelian Randomization analysis to uncover

389 novel mechanisms that contribute towards disease susceptibility ${ }^{88-91}$.

390 A main application for ExPRSs might be their use as proxies for unmeasured

391 exposures. Exposures relevant for many conditions are often only sparsely measured in

392 the EHR data sets and their missingness can substantially reduce sample size when

393 considering only complete case datasets (as seen here for PXS). Furthermore, contrary

394 to genotype data the missingness can be non-random because testing generally is

395 selective, diagnosis- and symptom-specific, as seen here for 9 of the 12 analyzed

396 conditions, and thus likely would bias prediction models. Nevertheless, an ExPRS can

397 even in the best scenario only capture the heritable fraction of the exposure's variance

398 coming from variants assigned at birth but not the lifelong exposure to environmental or

399 consequences of behavioral factors. Using ExPRSs for the imputation of incomplete

400 exposure data could be worth further explorations but was not within the scope of the

401 current study.

402 Being dependent on large GWAS and evaluation cohorts we expect that future

403 studies will provide more powerful YPRS and ExPRSs. But even then, the interplay of

404 genetic and non-genetic factors needs to be considered when assessing complex traits.

405 Current large biobank efforts link genotype data with EHRs, and often complement

406 patient information on environmental, lifestyle, and demographic variables via self-report 
medRxiv preprint doi: https://doi.org/10.1101/2022.01.13.22269176; this version posted January 14, 2022. The copyright holder for this preprint

(which was not certified by peer review) is the author/funder, who has granted medRxiv a license to display the preprint in perpetuity.

It is made available under a CC-BY-NC-ND 4.0 International license.

$407 \quad 92$. The integration of these resources will likely improve our models with the goal to

408 prevent or treat conditions earlier.

$409 \quad$ Finally, we created an online repository called "ExPRSweb" that similar to our

410 cancer specific PRS repository "Cancer PRSweb" ${ }^{20}$, provides an interactive platform to

411 browse performance metrics of all generated ExPRSs in two independent biobanks. We

412 also deposited all promising ExPRSs to the PGS catalog and linked it to ExPRSweb

413 and our evaluations. We anticipate that ExPRSweb can serve as an example and a

414 standardized platform to expedite ExPRS research and to facilitate easier access. 


\section{Methods}

\section{Michigan Genomics Initiative (MGI)}

\section{MGI Cohort}

Adult participants aged between 18 and 101 years at enrollment were recruited through

421 the Michigan Medicine health system between 2012 and 2020. Participants have

422 consented to allow research on both their biospecimens and EHR data, as well as

423 linking their EHR data to national data sources such as medical and pharmaceutical

424 claims data. Participants were primarily recruited through the MGI - Anesthesiology

425 Collection Effort while awaiting a diagnostic or interventional procedure either at a

426 preoperative appointment or on the day of their operative procedure at Michigan

427 Medicine. Additional participants were recruited through the Michigan Predictive Activity 428 and Clinical Trajectories (MIPACT) Study, the Mental Health BioBank (MHB2), and the 429 Michigan Genomics Initiative-Metabolism, Endocrinology, and Diabetes (MGI-MEND)

430 Study. The data used in this study included diagnoses coded with the Ninth and Tenth

431 Revision of the International Statistical Classification of Diseases (ICD9 and ICD10) with

432 clinical modifications (ICD9-CM and ICD10-CM), laboratory measurements,

433 anthropometrics (height, thinness and body mass index [BMI]), vitals (systolic and

434 diastolic blood pressure [SBP and DBP]), health behavior (alcohol amount, smoker and

435 drinker), sex, precomputed principal components (PCs), genotyping batch, recruitment

436 study, and age. Data were collected according to the Declaration of Helsinki principles.

437 MGI study participants' consent forms and protocols were reviewed and approved by

438 the University of Michigan Medical School Institutional Review Board (IRB ID 
439 HUM00099605 and HUM00155849). Opt-in written informed consent was obtained.

440 Additional details about MGI can be found online (see Web Resources).

\section{MGI Genotype Data}

443 DNA from 56,984 blood samples was genotyped on customized Illumina Infinium

444 CoreExome-24 bead arrays and subjected to various quality control filters, resulting in a

445 set of 502,255 polymorphic variants. Principal components and ancestry were estimated

446 by projecting all genotyped samples into the space of the principal components of the

447 Human Genome Diversity Project reference panel using PLINK (v1.90b5.4, 938

448 individuals) ${ }^{93,94}$. Pairwise kinship was assessed with the software KING (2.2.4) ${ }^{95}$, and

449 the software Fastlndep was used to reduce the data to a maximal subset that contained

450 no pairs of individuals with 3rd-or closer degree relationship ${ }^{96}$. We removed participants

451 without EHR data and participants not of recent European descent from the analysis,

452 resulting in a final sample of 46,782 unrelated subjects. Additional genotypes were

453 obtained using the Haplotype Reference Consortium reference panel of the Michigan

454 Imputation Server ${ }^{97}$ and included over 24 million imputed variants with $\mathrm{R}^{2} \geq 0.3$ and

455 minor allele frequency (MAF) $\geq 0.01 \%$.

\section{MGl Phenome}

458 The MGI phenome was based on ICD9-CM and ICD10-CM code data for 46,782

459 unrelated, genotyped individuals of recent European ancestry. Longitudinal time-

460 stamped diagnoses were recoded to indicators for whether a patient ever had given a

461 diagnosis code recorded by Michigan Medicine. These ICD9-CM and ICD10-CM codes

462 were aggregated to form up to 1,814 PheCodes using the PheWAS R package (as 
463 described in detail elsewhere $20,98,99)$. To minimize differences in age and sex

464 distributions, avoid extreme case-control ratios, and reduce the computational burden,

465 we matched up to 10 controls to each case using the R package "Matchlt" ${ }^{100}$. Nearest

466 neighbor matching was applied for age and the first four principal components of the

467 genotype data (PC1-4) using Mahalanobis distance with a caliper/width of 0.25 standard

468 deviations. Exact matching was applied for sex and genotyping array. A total of 1,685

469 case-control studies with $>50$ cases were used for our analyses of the MGl phenome.

470

471 MGI Common Chronic Conditions

472 We used the CCW Condition Algorithms (rev. 02/2021) from the CMS Chronic Condition

473 Warehouse (CCW; see Web Resources) to define 27 common chronic conditions in

474 MGI. In short, like the PheCode system the CCW algorithms are based on ICD-9-CM-

475 and ICD-10-CM-based inclusion and exclusion criteria. Here, we were interested in any

476 observation of such conditions and disregarded the algorithms' stated reference period

477 or the required numbers/types of qualifying claims for Medicare or Medicaid. The

478 resulting 27 case-control studies were labelled CCW01-CCW27 and are listed in

479 Table S12.

480

481 UK Biobank cohort (UKB).

482

483 UKB Cohort

$484 \quad$ UKB is a population-based cohort collected from multiple sites across the United

485 Kingdom and includes over 500,000 participants aged between 40 and 69 years when 
486 recruited in 2006-2010 ${ }^{101}$. The open-access UK Biobank data used in this study

487 included genotypes, ICD9 and ICD10 codes, biomarker data, anthropometrics, vitals,

488 women's health, health behavior, inferred sex, inferred White British ancestry, kinship

489 estimates down to third degree, birthyear, genotype array, and precomputed principal

490 components of the genotypes. UK Biobank received ethical approval from the NHS

491 National Research Ethics Service North West (11/NW/0382).

492

493

494

495

UKB Genotype Data

496408,595 White British 102 individuals and 47,836,001 variants with imputation

497 information score $>=0.3$ and MAF $\geq 0.01 \%$ of which $22,933,317$ overlapped with the

498 imputed MGI data (see above). Two random subsets of 5,000 and 10,000 unrelated,

499 White British individuals were used for LD analyses of UKB-based summary statistics.

500 Genotyping, quality control, and imputation are described in detail elsewhere ${ }^{98}$.

501

502

503

504 The UK Biobank phenome was based on ICD9 and ICD10 code data of 408,595 White

505 British ${ }^{102}$, genotyped individuals that were similarly aggregated to PheCodes as MGI

506 (see above, also described elsewhere ${ }^{103}$ ). In contrast to MGI, there were many pairwise

507 relationships reported for UKB participants.

508 To retain a larger effective sample size for each phenotype, we first selected a maximal

509 set of unrelated cases for each phenotype (defined as no pairwise relationship of $3^{\text {rd }}$

510 degree or closer ${ }^{96,104}$ ) before selecting a maximal set of unrelated controls unrelated to

511 these cases. Similar to MGI, we matched up to 10 controls to each case using the $R$ 
512 package "Matchlt" ${ }^{100}$. Nearest neighbor matching was applied for birthyear (as proxy for

513 age because age at diagnosis was not available to us) and PC1-4 (Mahalanobis-metric

514 matching; matching window caliper/width of 0.25 standard deviations), and exact

515 matching was applied for sex and genotyping array. A total of 1,419 matched case-

516 control studies with $>50$ cases each were used for our analyses of the UK Biobank

517 phenome.

519 Exposure data

520 For a set of 21 continuous and 7 binary exposures for which we could find freely

521 available and complete GWAS summary statistics (see Exposure GWAS Summary

522 Statistics below) we extracted the corresponding EHR data as described in Table S1.

523 For the binary exposures that are common disorders (type 2 diabetes, hypertension,

524 insomnia, and sleep apnea) we use the PheWAS code-based definitions (see MGI and

525 UKB phenome above; Table S7). Survey based measures with multiple responses per

526 person (never/past/current alcohol use and smoking status) were recoded to never /

527 ever responses. Continuous exposures with multiple measurements across multiple

528 visits (e.g., BMI, biomarkers, or blood pressure values) were harmonized as follows: we

529 first removed outliers per individual per trait by using the interquartile range (IQR)

530 criterion. Next, we aggregated multiple entries per individual by using the mean value

531 across multiple entries per person per trait as the value of the corresponding continuous

532 trait. For the UKB cohort, we calculated the estimated Glomerular Filtration Rate

533 (eGFR) on the natural scale using the harmonized serum creatinine values (data field 
534 30700), race and sex information and the Chronic Kidney Disease Epidemiology

535 Collaboration (CKD-EPI) equation ${ }^{105}$.

\section{Exposure GWAS Summary Statistics}

538 For each of the 28 exposures, we collected complete GWAS summary statistics from up

539 to four different sources: (1) catalogued GWAS of the NHGRI EBI GWAS Catalog ${ }^{106}$ (2)

540 GWAS from the FinnGen Consortium; (3) published GWAS meta-analyses; and (4)

541 publicly available GWAS summary statistics of phenome $x$ genome screening efforts of

542 the UK Biobank data (Lee and Neale Lab, see Table S2 and Web Resources). We

543 only included GWAS summary statistics of studies that analyzed broad European

544 ancestry to match the ancestry of discovery GWAS and target cohorts (MGI and UKB).

545 If needed, we lifted over coordinates of GWAS summary statistics to human genome

546 assembly GRCh37 (LiftOver, UCSC Genome Browser Store, see Web Resources).

547 Entries with missing effect alleles, or effect sizes were excluded. If effect allele

548 frequency (EAF) was reported in the summary statistics, we also compared EAF

549 between the discovery GWAS and the target dataset (MGI and/or UKB). If the

550 proportion of likely flipped alleles (whose RAF deviated more than $15 \%$ between the

551 datasets) was above $40 \%$ we excluded the GWAS as source for PRS construction.

552 These chosen thresholds were subjective and based on clear differentiation between

553 correct and likely flipped alleles on the two diagonals, as noted frequently in GWAS

554 meta-analyses quality control procedures.

555

\section{Statistical Methods}




\section{Heritability Estimation}

558 For each set of GWAS summary statistics from both UK Biobank and non-UK Biobank

559 sources (e.g., FinnGen, GWAS catalog, large meta-analyses), we first estimated the

560 SNP heritability to estimate the proportion of phenotypic variance explained by all

561 measured SNPs based on summary statistics. The estimated SNP heritability

562 represents the upper limit for the prediction performance of PRS methods and serves as

563 an initial filtering criterion to validate the quality of the downloaded summary statistics.

564 To do so, we applied the method MQS (MinQue for Summary statistics), which was

565 implemented in Gemma, to calculate the SNP heritability estimate (see Web

566 Resources) ${ }^{107,108}$. MQS estimates the SNP heritability based on the Minimal Norm

567 Quadratic Unbiased Estimation (MINQUE) ${ }^{109,110}$ criterion. Specifically, we first converted

568 the $p$-values into marginal z-scores, then we used the $z$-scores as well as 5,000

569 randomly selected, unrelated samples (reference panel) as input to run Gemma. Finally,

570 we obtained the proportion of variance in phenotypes explained (PVE) estimates from

571 Gemma which corresponds to the SNP heritability estimate. We further filtered out the

572 summary statistics that had negative heritability estimates.

573 For binary traits with potentially ascertained case-control data, we converted the

574 heritability estimates from the observed scale to the liability scale using the R package

575 "PDRohde/ugnome" and reported population prevalence estimates (Table S3) ${ }^{111 .}$

576

577 ExPRS Construction

578 We constructed the PRS for an individual $\mathrm{j}$ in the form $\mathrm{PRS} S_{j}=\sum_{i} \beta_{i} G_{i j}$ where $i$ indexes the 579 included variants for that trait, weight $\beta_{i}$ is the log odds ratios retrieved from the external 
580 GWAS summary statistics for variant $i$, and $G_{i j}$ is a continuous version of the measured

581 dosage data for the risk allele of variant $i$ in subject $j$. To construct a PRS, one must

582 determine which genetic loci to include in the PRS and their relative weights. We have

583 obtained GWAS summary statistics from several external sources, resulting in several

584 sets of weights for each trait of interest. For each set of weights obtained from GWAS

585 summary statistics from the above-mentioned sources and each trait, we generated for

586 each exposure GWAS up to 5 different PRSs reflecting the 5 implementations of 4

587 different PRS methods: the $\mathrm{C}+\mathrm{T}$ (both, best guess genotype [GT] and dosage [DS]

588 version) ${ }^{25-27}$, lassosum ${ }^{28}$, DBSLMM $^{29}$ and PRS-CS ${ }^{30}$ (Figure 1).

589 We summarized the statistical aspects of these construction methods in Table S22. The

590 goal of this approach was to compare multiple PRS methods and find the method that

591 works best for the various types of GWAS summary statistics.

592 LD clumping and P-value Thresholding $(C+T)$

593 We performed linkage disequilibrium (LD) clumping/pruning of variants with $p$-values

594 below 0.1 by using the imputed allele dosages of 10,000 randomly selected samples

595 and a pairwise correlation cut-off at $\mathrm{r}^{2}<0.1$ within $1 \mathrm{Mb}$ window. We constructed many

596 different PRS across a fine grid of $p$-value thresholds. The $p$-value threshold with the

597 highest pseudo- $\mathrm{R}^{2}$ (binary trait) or highest $\mathrm{R}^{2}$ (continuous traits) (see PRS Evaluation

598 below) was used to define the optimized "Clumping and Thresholding (C \& S)" PRS. We

599 applied two approaches for LD clumping: $\mathrm{C}+\mathrm{T}(\mathrm{GT})$ and $\mathrm{C}+\mathrm{T}(\mathrm{DS})$. Specifically, the " $\mathrm{C}$

$600+\mathrm{T}(\mathrm{GT})$ )" is implemented by plink-1.9 using the best guess genotypes (GT, imputed

601 genotype dosages are rounded to the next integer) for LD calculations, while "C+T (DS)" 
medRxiv preprint doi: https://doi.org/10.1101/2022.01.13.22269176; this version posted January 14, 2022. The copyright holder for this preprint (which was not certified by peer review) is the author/funder, who has granted medRxiv a license to display the preprint in perpetuity.

It is made available under a CC-BY-NC-ND 4.0 International license .

602 is implemented in R and considers the uncertainty of imputed genotypes by using the

603 dosage data (DS).

604 Lassosum

605 Lassosum obtains PRS weights by applying elastic net penalization to GWAS summary

606 statistics and incorporating LD information from a reference panel. Here, we used 5,000

607 randomly selected, unrelated samples as the LD reference panel. We applied a MAF

608 filter of $1 \%$ and, in contrast to the previous two approaches, only included autosomal

609 variants that overlap between summary statistics, LD reference panel, and target panel.

610 Each "Lassosum" run resulted in up to 76 combinations of the elastic net tuning

611 parameters $s$ and $\lambda$, and consequently, in 76 SNP sets with corresponding weights used

612 to construct. We then selected the PRS with the pseudo- $R^{2}$ (binary trait) or highest $R^{2}$

613 (continuous traits) to define the "Lassosum" PRS (see PRS Evaluation below).

614 Deterministic Bayesian Sparse Linear Mixed Model (DBSLMM)

615 DBSLMM assumes that the true SNP effect sizes derive from a mixture of normal

616 distributions and relies on an efficient deterministic search algorithm for statistical

617 inference. DBSLMM requires both GWAS summary statistics and LD information from a

618 reference panel. Specifically, DBSLMM first selects SNPs with large effect in a

619 deterministic fashion through the $\mathrm{C}+\mathrm{T}$ procedure and then directly obtains both large

620 SNP effect sizes and small SNP effect sizes through analytic forms. Here, we used

6215,000 randomly selected unrelated samples as the LD reference panel. We applied a

622 MAF filter of $1 \%$ and only included autosomal variants that overlap between summary

623 statistics, LD reference panel, and target panel. Heritability estimates obtained from

624 Gemma (see above-mentioned procedure) were used as the input of DBSLMM. All 
625 other parameters we used are the default parameters in the "DBSLMM" software. For

626 example, we set the cutoff of SNPs clumping and pruning to be $r^{2}<0.1$ within a $1 \mathrm{Mb}$

627 window and $p$-value $<1 \mathrm{e}-06$, respectively. Each DBSLMM run resulted in one SNP set

628 with corresponding weights to construct the PRS. We used the default version of

629 DBSLMM which does not require cross-validation and refer to the obtained PRS as

630 “DBSLMM" PRS.

$631 \quad P R S-C S$

632 PRS-CS utilizes a Bayesian regression framework and assumes a continuous

633 shrinkage (CS) prior on the effect sizes. Specifically, we applied the default "auto"

634 version of PRS-CS that obtain weights through Gibbs sampling algorithm. Here, PRS-

635 CS-auto uses a precomputed LD reference panel based on external European samples

636 of the 1000 Genomes Project ("EUR reference") to construct a PRS. We applied a MAF

637 filter of $1 \%$ and only included autosomal variants that overlap between summary

638 statistics, LD reference panel, and target panel. The obtained PRS is referred to as

639 "PRS-CS" PRS.

640 For each trait and set of GWAS summary statistics, these approaches usually

641 resulted in up to five PRS. However, approaches that resulted in less than 5 weights /

642 variants were excluded. Using the R package "Rprs" (see Web Resources), the value

643 of each PRS was then calculated for each MGI participant and, if the GWAS source did

644 to the best of our knowledge did not include UKB samples, also for each UKB

645 participant. For comparability of association effect sizes corresponding to the

646 continuous PRS across exposures and PRS construction methods, we centered PRS 
647 values in MGI and UKB to a mean of 0 and scaled them to have a standard deviation of

6481.

\section{ExPRS Evaluation}

651 To assess the predictive performance of these generated PRS, each PRS was

652 assessed through cross-validation in either the MGI cohort or the UKB cohort: we split

653 the data corresponding to each trait in training $(50 \%$ of the samples with gender ratio

654 unchanged) and test set (50\% of the samples with gender ratio unchanged). We used

655 the training set to determine the PRS tuning parameter(s) and used the testing set to

656 obtain performance metric for that PRS.

657 For the PRS evaluations, except for when computing the pseudo- $R^{2}$ for binary

658 exposures (which is a measure of marginal association of the ExPRS with the

659 exposure $)^{112}$, we fit the following model for each PRS and exposure adjusting for

660 covariates:

$661 \quad \mathrm{~g}(\mathrm{E}($ Exposure $\mid \mathrm{PRS}$, Age, Sex, Array, PCs $))=\beta_{0}+\beta_{P R S} \mathrm{PRS}+\beta_{\text {Age }}$ Age +

$662 \beta_{\text {Sex }}$ Sex $+\beta_{\text {Array }}$ Array $+\beta_{P C S}$ PCs,

663 where $g(\cdot)$ is the link function (e.g., identity link function for continuous traits and logit

664 link function for binary traits). PCs were the first four principal components obtained

665 from the principal component analysis of the genotyped GWAS markers, where "Age"

666 was the age at last observed diagnosis in MGI and birthyear in UKB and where "Array"

667 represents the genotyping array.

668 Binary Traits 
669 We used Nagelkerke's pseudo- $\mathrm{R}^{2}$ to select the tuning parameters within the " $\mathrm{C}+\mathrm{T}$ " and

670 Lassosum construction methods ( $\mathrm{P}$-value for " $\mathrm{C}+\mathrm{T}$ " SNP sets; s and $\lambda$ for Lassosum)

671 and kept the PRS with the highest pseudo- $\mathrm{R}^{2}$ for further analyses. For each PRS

672 derived for each GWAS source/method combination, we assessed the following

673 performance measures relative to observed disease status in MGI and UKB: (1) overall

674 performance with Nagelkerke's pseudo-R² using R packages “rcompanion”, (2)

675 accuracy with Brier score using R package "DescTools"; and (3) ability to discriminate

676 between cases and controls as measured by the area under the covariate-adjusted

677 receiver operating characteristic (AROC; semiparametric frequentist inference) curve

678 (denoted AAUC) using R package "ROCnReg". Firth's bias reduction method was used

679 to resolve the problem of separation in logistic regression (R package "brglm2").

680 Continuous Traits

681 For the PRS evaluations of continuous traits, we used $\mathrm{R}^{2}$ to select the tuning

682 parameters within the " $\mathrm{C}+\mathrm{T}$ " and Lassosum construction methods (P-value for " $\mathrm{C}+\mathrm{T}$ "

683 SNP sets; s and $\lambda$ for Lassosum) and kept the PRS with the highest $\mathrm{R}^{2}$ for further

684 analyses. For each PRS derived for each GWAS source/method combination, we

685 assessed the prediction performance in terms of $R^{2}$ in MGI and UKB.

686

687 ExPRS Primary Association with the Underlying Exposure

688 Next, we assessed the strength of the relationship between these PRSs and the traits

689 they were designed for. To do this, we fit the same model as equation (1). Our primary

690 interest is $\beta_{P R S}$, while the other factors (Age, Sex and PCs) were included to address

691 potential residual confounding. Firth's bias reduction method was used to resolve the 
692 problem of separation in logistic regression (Logistf in R package "EHR"). As an initial

693 filtering step, we removed PRS that were not significantly associated with their

694 corresponding exposure in MGI or UKB cohort $(P>0.05)$ for downstream analysis. The

695 majority of these filtered PRS were either based on discovery GWAS with small sample

696 sizes that often did not identify any genome-wide significant hits or were evaluated for

697 exposure with small sample sizes or both, indicating a potential lack of power in our 698 analysis.

699

700 Illustrative Examples showcasing the use of ExPRS

701 Once we select the ExPRS that were mostly and positively associated with the specific

702 exposure, referred to as the best performing PRS, we use these selected PRSs for

703 various analyses to illustrate how a user may gainfully use these constructs in

704 understanding disease etiology and mechanisms.

705 Phenome-wide Exploration of ExPRS Associations

706 We conducted PheWAS in MGI and UKB (if the GWAS source was not based on

707 UKB) to identify phenotypes associated with the ExPRS. To evaluate ExPRS-phenotype

708 associations, we conducted Firth bias-corrected logistic regression by fitting the

709 following model for each ExPRS and each phenotype of the corresponding phenome.

710 logit $(\mathrm{P}($ Phecode is present | ExPRS, Age, Sex, Array, PCs $))=\beta_{0}+$

$711 \beta_{\text {ExPRS }}$ ExPRS $+\beta_{\text {Age }}$ Age $+\beta_{\text {Sex }}$ Sex $+\beta_{\text {Array }}$ Array $+\beta_{P C S}$ PCs

712 To adjust for multiple testing, we applied the conservative phenome-wide

713 Bonferroni correction according to the total number of analyzed PheCodes (MGI: 1,685

714 phenotypes; UKB: 1,419 phenotypes as described in Table S7). In Manhattan plots, we 
715 present $-\log 10$ ( $p$-value) corresponding to tests for association of the underlying

716 phenotype with the ExPRS. Directional triangles on the PheWAS plot indicate whether a

717 trait was positively (pointing up) or negatively (pointing down) associated with the

718 ExPRS.

719 To investigate the possibility of the secondary trait associations with ExPRS

720 being completely driven by the exposure or extremes of the trait distribution, we

721 performed a second set of PheWAS: for binary exposures we excluded individuals with

722 the binary exposures for which the ExPRS was constructed; for continuous exposures

723 we excluded individuals with measurements outside of the normal range (Table S1).

724 We referred to these PheWAS as "Exclusion-PRS-PheWAS" as described previously ${ }^{33}$.

725 To evaluate whether the constructed ExPRS is a good proxy for the

726 corresponding exposure, we also repeated the PheWAS using the exposure or normal

727 range exposure as the predictor instead. We referred to these PheWAS as "Trait-

728 PheWAS" and "Exclusion-Trait-PheWAS", respectively.

729

730 Utilities of ExPRS on Common Chronic Conditions

731 To investigate the utility of our constructed ExPRS in predicting Common Chronic

732 Condition in the MGI cohort (see MGI Common Chronic Conditions above, Table S12).

733 We first split the common chronic conditions into training $(50 \%$ of the samples with

734 gender ratio unchanged) and test set (50\% of the samples with gender ratio

735 unchanged). We conducted Firth bias-corrected logistic regression by fitting the

736 following model for each of the best performing ExPRS and each common chronic

737 condition: 
$740 \quad$ Prediction performance was measured by Nagelkerke's pseudo- $R^{2}$, Brier score

741 and AAUC. Then we repeated the analysis using the actual exposure as predictor to be

742 trained and evaluated in the MGI cohort.

$743 \quad$ Next, we selected for each chronic condition the ExPRSs that reached nominal

744 significance in the univariate model and performed clumping $(r<0.5)$. For each chronic

745 condition, we combined the resulting sets of their associated ExPRSs by fitting a logistic

746 regression in the training set to obtain the linear predictors that we defined as

747 "multiExPRS" in the testing data.

748 To investigate whether such multiExPRSs can be helpful in predicting a common

749 chronic condition "Y" beyond the condition specific PRS "YPRS" (e.g., breast cancer

750 PRS), we collected the YPRS from public resources, except for type 2 diabetes and

751 hypertension for which we generated ExPRS. We downloaded PRS constructs/weights

752 for lung cancer, prostate cancer, colorectal cancer and breast cancer PRS from Cancer

753 PRSweb ${ }^{113}$ (https://prsweb.sph.umich.edu) and downloaded the following PRS weight

754 from the PGS Catalog ${ }^{114}$ (https://www.pgscatalog.org): Stroke/Transient Ischemic

755 Attach, Heart Failure, Glaucoma, Chronic Kidney Disease, Atrial Fibrillation and Asthma

756 PRS. We harmonized the downloaded PRS weights to GRCh37/hg19 and determined

757 overlap with the MGI genotype data. Non-ambiguous SNP alleles were flipped to the

758 genomic plus strand. We fit three logistic models for each common chronic condition " $Y$ "

759 using the following predictors adjusting for the set of covariates from above: (1)

760 condition specific PRS "YPRS", (2) the combined ExPRS "multiExPRS", and (3) 
761 "multiExPRS + YPRS". As before, we combined multiple predictors fitting a logistic

762 regression in the training set to obtain the linear predictors that we used as combined

763 score in the testing data.

$764 \quad$ To study the ability of these three predictors to identify high risk patients for these

765 chronic conditions, we fit the three multi-variate logistic model but replaced the risk

766 scores with an indicator for whether the risk scores value was in the top $1,2,5,10$, or

$76725 \%$ (defined in controls) of the risk score distribution.

768

769 Poly-Exposure Score Construction and Comparison

770 To contrast the predictive power of a poly-exposure score (PXS) with combined

771 ExPRSs (multiExPRS, see above), we extracted the collected / measured exposure

772 data from MGI. We removed three exposures (Cystatin C, Fasting Plasma Glucose,

773 Estradiol levels) that due to their high missingness would have led to very small sample

774 sizes in a complete case analysis across multiple exposures.

775 We retained the training / testing data split from the "ExPRS Evaluation" (see above)

776 and ran the following model for each of the remaining exposures and each of the

777 selected common chronic conditions in the training data:

778 logit ( $\mathrm{P}($ a common chronic condition is present | Exposure, Age, Sex, Array, PCs))

$779=\beta_{0}+\beta_{\text {Exposure }}$ Exposure $+\beta_{\text {Age }}$ Age $+\beta_{\text {Sex }}$ Sex $+\beta_{\text {Array }}$ Array $+\beta_{P C S}$ PCs,

780

781 Similar to the multiExPRSs, we selected the significantly associated exposures and

782 performed clumping to only retain the significantly associated exposures with a

783 correlation $<0.5$ with each other. The remaining set of exposures was used to create a 
784 complete case training data set that we used to obtain effect sizes for each exposure

785 that we used as weights to create weighted exposures in the complete case testing

786 data. The weighted exposures were then combined into a single predictor that we refer

787 to as poly-exposure score (PXS). Finally, we compared the AAUC of following four

788 predictors adjusting for the set of covariates from above: the condition specific PRS

789 ("YPRS"), the combined ExPRSs "multiExPRS", the "multiExPRS + YPRS" and the

790 PXS.

791

792 Online Visual Catalog: ExPRSweb

793 The online open access visual catalog ExPRSweb (see Web Resources) was

794 implemented using Grails as previously described ${ }^{20}$.

795

796 Unless otherwise stated, analyses were performed using R 4.1.1. We used the

797 STREGA checklist when writing our report ${ }^{115}$.

798

799 


\section{Acknowledgement}

801 The authors acknowledge the Michigan Genomics Initiative participants, Precision

802 Health at the University of Michigan, and the University of Michigan Medical School

803 Data Office for Clinical and Translational Research, the University of Michigan Medical

804 School Central Biorepository, and the University of Michigan Advanced Genomics Core

805 for providing data storage, management, processing, and distribution services, and the

806 Center for Statistical Genetics in the Department of Biostatistics at the School of Public

807 Health for genotype data curation, imputation, and management in support of the

808 research reported in this publication.

809 We would like to thank Alison Mondul and Brett Vanderwerff (University of Michigan

810 School of Public Health) for careful reading of the manuscript.

811 Part of this research has been conducted using both the UK Biobank Resource under

812 application number 24460 and using results and data generated by previous

813 researchers who have used the UK Biobank Resource.

814 This material is based in part upon work supported by the National Institutes of

815 Health/NIH (NCI P30CA046592 [LGF, BM]), by the University of Michigan (UM-

816 Precision Health Investigators Award U063790 [LGF, SP, YM, BM]), and by the National

817 Science Foundation under grant number DMS-1712933. Any opinions, findings, and

818 conclusions or recommendations expressed in this material are those of the author(s)

819 and do not necessarily reflect the views of the National Science Foundation. 
medRxiv preprint doi: https://doi.org/10.1101/2022.01.13.22269176; this version posted January 14, 2022. The copyright holder for this preprint (which was not certified by peer review) is the author/funder, who has granted medRxiv a license to display the preprint in perpetuity.

\section{References}

822 1. Buniello, A. et al. The NHGRI-EBI GWAS Catalog of published genome-wide 823 association studies, targeted arrays and summary statistics 2019. Nucleic Acids

2. Genin, E. Missing heritability of complex diseases: case solved? Hum Genet 139, 103-113 (2020).

3. Manolio, T.A. et al. Finding the missing heritability of complex diseases. Nature 461, 747-53 (2009).

4. Yang, J. et al. Common SNPs explain a large proportion of the heritability for human height. Nature genetics 42, 565-569 (2010).

5. Kamps, R. et al. Next-Generation Sequencing in Oncology: Genetic Diagnosis, Risk Prediction and Cancer Classification. Int J Mol Sci 18(2017).

6. Jostins, L. \& Barrett, J.C. Genetic risk prediction in complex disease. Hum Mol Genet 20, R182-8 (2011).

7. Ma, Y. \& Zhou, X. Genetic prediction of complex traits with polygenic scores: a statistical review. Trends Genet 37, 995-1011 (2021).

8. Meigs, J.B. et al. Body mass index, metabolic syndrome, and risk of type 2 diabetes or cardiovascular disease. J Clin Endocrinol Metab 91, 2906-12 (2006).

9. Almirall, J., Serra-Prat, M., Bolíbar, I. \& Balasso, V. Risk factors for communityacquired pneumonia in adults: a systematic review of observational studies. Respiration 94, 299-311 (2017).

10. Pierce, B.L., Kraft, P. \& Zhang, C. Mendelian randomization studies of cancer risk: a literature review. Curr Epidemiol Rep 5, 184-196 (2018).

11. Kachuri, L. et al. Pan-cancer analysis demonstrates that integrating polygenic risk scores with modifiable risk factors improves risk prediction. Nat Commun 11, 6084 (2020).

12. Haneuse, S. Distinguishing Selection Bias and Confounding Bias in Comparative Effectiveness Research. Med Care 54, e23-9 (2016).

13. Beesley, L.J. \& Mukherjee, B. Statistical inference for association studies using electronic health records: handling both selection bias and outcome misclassification. Biometrics (2020).

14. Loos, R.J.F. 15 years of genome-wide association studies and no signs of slowing down. Nat Commun 11, 5900 (2020).

15. Liu, M. et al. Association studies of up to 1.2 million individuals yield new insights into the genetic etiology of tobacco and alcohol use. Nat Genet51, 237-244 (2019).

16. Khera, A.V. et al. Genome-wide polygenic scores for common diseases identify individuals with risk equivalent to monogenic mutations. Nat Genet 50, 1219-1224 (2018).

17. Lambert, S.A., Abraham, G. \& Inouye, M. Towards clinical utility of polygenic risk scores. Hum Mol Genet 28, R133-R142 (2019).

18. Tam, C.H.T. et al. Development of genome-wide polygenic risk scores for lipid traits and clinical applications for dyslipidemia, subclinical atherosclerosis, and diabetes cardiovascular complications among East Asians. Genome Med 13, 29 (2021). 
medRxiv preprint doi: https://doi.org/10.1101/2022.01.13.22269176; this version posted January 14 , 2022. The copyright holder for this preprint (which was not certified by peer review) is the author/funder, who has granted medRxiv a license to display the preprint in perpetuity. It is made available under a CC-BY-NC-ND 4.0 International license .

19. Ma, Y. \& Zhou, X. Genetic prediction of complex traits with polygenic scores: a statistical review. Trends Genet (2021).

20. Fritsche, L.G. et al. Cancer PRSweb: An Online Repository with Polygenic Risk Scores for Major Cancer Traits and Their Evaluation in Two Independent Biobanks. Am J Hum Genet 107, 815-836 (2020).

21. Andrews, S.J. et al. Causal Associations Between Modifiable Risk Factors and the Alzheimer's Phenome. Ann Neurol 89, 54-65 (2021).

22. Li, S. \& Schooling, C.M. A phenome-wide association study of genetically mimicked statins. BMC Med 19, 151 (2021).

23. Richardson, T.G., Harrison, S., Hemani, G. \& Davey Smith, G. An atlas of polygenic risk score associations to highlight putative causal relationships across the human phenome. Elife 8(2019).

24. Lambert, S.A. et al. The Polygenic Score Catalog as an open database for reproducibility and systematic evaluation. Nat Genet 53, 420-425 (2021).

25. Wray, N.R., Goddard, M.E. \& Visscher, P.M. Prediction of individual genetic risk to disease from genome-wide association studies. Genome Res 17, 1520-8 (2007).

26. International Schizophrenia, C. et al. Common polygenic variation contributes to risk of schizophrenia and bipolar disorder. Nature 460, 748-52 (2009).

27. Euesden, J., Lewis, C.M. \& O'Reilly, P.F. PRSice: Polygenic Risk Score software. Bioinformatics 31, 1466-8 (2015).

28. Mak, T.S.H., Porsch, R.M., Choi, S.W., Zhou, X. \& Sham, P.C. Polygenic scores via penalized regression on summary statistics. Genet Epidemiol 41, 469-480 (2017).

29. Yang, S. \& Zhou, X. Accurate and Scalable Construction of Polygenic Scores in Large Biobank Data Sets. Am J Hum Genet 106, 679-693 (2020).

30. Ge, T., Chen, C.Y., Ni, Y., Feng, Y.A. \& Smoller, J.W. Polygenic prediction via Bayesian regression and continuous shrinkage priors. Nat Commun 10, 1776 (2019).

31. Sudlow, C. et al. UK biobank: an open access resource for identifying the causes of a wide range of complex diseases of middle and old age. PLoS medicine 12, e1001779 (2015).

32. Michigan Genomics Initiative (MGI). (2020).

33. Fritsche, L.G. et al. Association of polygenic risk scores for multiple cancers in a phenome-wide study: results from the Michigan Genomics Initiative. The American Journal of Human Genetics 102, 1048-1061 (2018).

34. Visscher, P.M., Hill, W.G. \& Wray, N.R. Heritability in the genomics era-concepts and misconceptions. Nature reviews genetics 9, 255-266 (2008).

35. Yang, J. et al. Genetic variance estimation with imputed variants finds negligible missing heritability for human height and body mass index. Nature genetics $\mathbf{4 7}$, 1114-1120 (2015).

36. Silventoinen, K. et al. Heritability of adult body height: a comparative study of twin cohorts in eight countries. Twin Research and Human Genetics 6, 399-408 (2003).

37. Johnson, D.A., Billings, M.E. \& Hale, L. Environmental determinants of insufficient sleep and sleep disorders: implications for population health. Current epidemiology reports 5, 61-69 (2018). 
medRxiv preprint doi: https://doi.org/10.1101/2022.01.13.22269176; this version posted January 14 , 2022. The copyright holder for this preprint (which was not certified by peer review) is the author/funder, who has granted medRxiv a license to display the preprint in perpetuity.

38. Ge, T., Chen, C.-Y., Ni, Y., Feng, Y.-C.A. \& Smoller, J.W. Polygenic prediction via Bayesian regression and continuous shrinkage priors. Nature communications $\mathbf{1 0}$, 1-10 (2019).

39. Vilhjálmsson, B.J. et al. Modeling linkage disequilibrium increases accuracy of polygenic risk scores. The american journal of human genetics 97, 576-592 (2015).

40. Kulm, S., Mezey, J. \& Elemento, O. Benchmarking the accuracy of polygenic risk

916 scores and their generative methods. MedRxiv 10, 06.20055574 (2020).

917

918

919

920

921

922

923

924

925

926

927

928

929

930

931

932

933

934

935

936

937

938

939

940

941

942

943

944

945

946

947

948

949

950

951

952

953

41. Pain, O. et al. Evaluation of polygenic prediction methodology within a referencestandardized framework. PLoS genetics 17, e1009021 (2021).

42. Ni, G. et al. A comparison of ten polygenic score methods for psychiatric disorders applied across multiple cohorts. Biological Psychiatry (2021).

43. Choi, S.W. \& O'Reilly, P.F. PRSice-2: Polygenic Risk Score software for biobankscale data. Gigascience 8(2019).

44. Chatterjee, N., Shi, J. \& Garcia-Closas, M. Developing and evaluating polygenic risk prediction models for stratified disease prevention. Nat Rev Genet 17, 392406 (2016).

45. Xue, A. et al. Genome-wide association analyses identify 143 risk variants and putative regulatory mechanisms for type 2 diabetes. Nature communications 9,1 14 (2018).

46. Liu, M. et al. Association studies of up to 1.2 million individuals yield new insights into the genetic etiology of tobacco and alcohol use. Nature genetics 51, 237-244 (2019).

47. Schillaci, G. \& Pucci, G. The dynamic relationship between systolic and diastolic blood pressure: yet another marker of vascular aging? Hypertension research 33, 659-661 (2010).

48. Gavish, B., Ben-Dov, I.Z. \& Bursztyn, M. Linear relationship between systolic and diastolic blood pressure monitored over $24 \mathrm{~h}$ : assessment and correlates. Journal of hypertension 26, 199-209 (2008).

49. Schillaci, G. \& Pucci, G. The dynamic relationship between systolic and diastolic blood pressure: yet another marker of vascular aging? Hypertens Res 33, 659-61 (2010).

50. Tam, C.H.T. et al. Development of genome-wide polygenic risk scores for lipid traits and clinical applications for dyslipidemia, subclinical atherosclerosis, and diabetes cardiovascular complications among East Asians. Genome medicine 13, 1-18 (2021).

51. Timpson, N.J. et al. C-reactive protein levels and body mass index: elucidating direction of causation through reciprocal Mendelian randomization. International journal of obesity 35, 300-308 (2011).

52. Unger, G., Benozzi, S.F., Perruzza, F. \& Pennacchiotti, G.L. Triglycerides and glucose index: a useful indicator of insulin resistance. Endocrinología y Nutrición (English Edition) 61, 533-540 (2014).

53. Beesley, L.J., Fritsche, L.G. \& Mukherjee, B. An analytic framework for exploring sampling and observation process biases in genome and phenome-wide association studies using electronic health records. Stat Med 39, 1965-1979 (2020). 
medRxiv preprint doi: https://doi.org/10.1101/2022.01.13.22269176; this version posted January 14 , 2022. The copyright holder for this preprint (which was not certified by peer review) is the author/funder, who has granted medRxiv a license to display the preprint in perpetuity. It is made available under a CC-BY-NC-ND 4.0 International license .

54. Farmer, R. et al. Promises and pitfalls of electronic health record analysis. Diabetologia 61, 1241-1248 (2018).

55. Gray, N., Picone, G., Sloan, F. \& Yashkin, A. The relationship between BMI and onset of diabetes mellitus and its complications. Southern medical journal 108, 29 (2015).

56. Wolk, R., Shamsuzzaman, A.S. \& Somers, V.K. Obesity, sleep apnea, and hypertension. Hypertension 42, 1067-1074 (2003).

57. Wolfe, B.M., Kvach, E. \& Eckel, R.H. Treatment of obesity: weight loss and bariatric surgery. Circulation research 118, 1844-1855 (2016).

58. Shivakumar, S., Srivastava, A. \& G, C.S. Body Mass Index and Dental Caries: A Systematic Review. Int J Clin Pediatr Dent 11, 228-232 (2018).

59. Coutinho, T. et al. Central obesity and survival in subjects with coronary artery disease: a systematic review of the literature and collaborative analysis with individual subject data. J Am Coll Cardiol 57, 1877-86 (2011).

60. Ng, R., Sutradhar, R., Yao, Z., Wodchis, W.P. \& Rosella, L.C. Smoking, drinking, diet and physical activity - modifiable lifestyle risk factors and their associations with age to first chronic disease. International journal of epidemiology 49, 113-130 (2020).

61. Wynder, E.L. et al. Screening for risk factors for chronic disease in children from fifteen countries. Preventive medicine 10, 121-132 (1981).

62. Chronic Conditions Data Warehouse. CCW Chronic Condition Categories. Vol. 2021.

63. $\mathrm{Xu}, \mathrm{W}$. et al. Meta-analysis of modifiable risk factors for Alzheimer's disease. $J$ Neurol Neurosurg Psychiatry 86, 1299-306 (2015).

64. Choi, S.W. \& O'Reilly, P.F. PRSice-2: Polygenic Risk Score software for biobankscale data. Gigascience 8, giz082 (2019).

65. He, Y. et al. Comparisons of Polyexposure, Polygenic, and Clinical Risk Scores in Risk Prediction of Type 2 Diabetes. Diabetes Care 44, 935-943 (2021).

66. Caldwell, M., Martinez, L., Foster, J.G., Sherling, D. \& Hennekens, C.H. Prospects for the Primary Prevention of Myocardial Infarction and Stroke. J Cardiovasc Pharmacol Ther 24, 207-214 (2019).

67. Reis, J.P. et al. Lifestyle factors and risk for new-onset diabetes: a populationbased cohort study. Ann Intern Med 155, 292-9 (2011).

68. Guilbert, J.J. The world health report 2002 - reducing risks, promoting healthy life. Educ Health (Abingdon) 16, 230 (2003).

69. Ellingjord-Dale, M. et al. Coffee consumption and risk of breast cancer: A Mendelian randomization study. Plos one 16, e0236904 (2021).

70. Grosso, G. et al. Coffee consumption and risk of all-cause, cardiovascular, and cancer mortality in smokers and non-smokers: a dose-response meta-analysis. (Springer, 2016).

71. $\mathrm{Xu}, \mathrm{J}$. et al. Leukocyte telomere length is associated with aggressive prostate cancer in localized prostate cancer patients. EBioMedicine 52, 102616 (2020).

72. Soleimani, A. et al. Role of the transforming growth factor - $\beta$ signaling pathway in the pathogenesis of colorectal cancer. Journal of cellular biochemistry 120, 8899-8907 (2019). 
medRxiv preprint doi: https://doi.org/10.1101/2022.01.13.22269176; this version posted January 14 , 2022. The copyright holder for this preprint (which was not certified by peer review) is the author/funder, who has granted medRxiv a license to display the preprint in perpetuity. It is made available under a CC-BY-NC-ND 4.0 International license .

1000

1001

1002

1003

1004

1005

1006

1007

1008

1009

1010

1011

1012

1013

1014

1015

1016

1017

1018

1019

1020

1021

1022

1023

1024

1025

1026

1027

1028

1029

1030

1031

1032

1033

1034

1035

1036

1037

1038

1039

1040

1041

1042

1043

1044

73. Kubiczkova, L., Sedlarikova, L., Hajek, R. \& Sevcikova, S. TGF- $\beta$-an excellent servant but a bad master. Journal of translational medicine 10, 1-24 (2012).

74. Wang, H., Peng, R., Wang, J., Qin, Z. \& Xue, L. Circulating microRNAs as potential cancer biomarkers: the advantage and disadvantage. Clinical epigenetics 10, 1-10 (2018).

75. Lloyd-Jones, L.R. et al. Improved polygenic prediction by Bayesian multiple regression on summary statistics. Nat Commun 10, 5086 (2019).

76. Prive, F., Arbel, J. \& Vilhjalmsson, B.J. LDpred2: better, faster, stronger. Bioinformatics (2020).

77. Chun, S. et al. Non-parametric Polygenic Risk Prediction via Partitioned GWAS Summary Statistics. Am J Hum Genet 107, 46-59 (2020).

78. Prive, F., Vilhjalmsson, B.J., Aschard, H. \& Blum, M.G.B. Making the Most of Clumping and Thresholding for Polygenic Scores. Am J Hum Genet 105, 12131221 (2019).

79. Marquez-Luna, C. et al. LDpred-funct: incorporating functional priors improves polygenic prediction accuracy in UK Biobank and 23andMe data sets. bioRxiv DOI: https://doi.org/10.1101/375337, 375337 (2020).

80. Hu, Y. et al. Leveraging functional annotations in genetic risk prediction for human complex diseases. PLoS Comput Biol 13, e1005589 (2017).

81. Maier, R. et al. Joint analysis of psychiatric disorders increases accuracy of risk prediction for schizophrenia, bipolar disorder, and major depressive disorder. Am J Hum Genet 96, 283-94 (2015).

82. Martin, A.R. et al. Human Demographic History Impacts Genetic Risk Prediction across Diverse Populations. Am J Hum Genet 100, 635-649 (2017).

83. Martin, A.R. et al. Clinical use of current polygenic risk scores may exacerbate health disparities. Nat Genet 51, 584-591 (2019).

84. Fritsche, L.G. et al. On Cross-ancestry Cancer Polygenic Risk Scores. medRxiv (2021).

85. Sirugo, G., Williams, S.M. \& Tishkoff, S.A. The Missing Diversity in Human Genetic Studies. Cell 177, 26-31 (2019).

86. Fritsche, L.G. et al. On Cross-ancestry Cancer Polygenic Risk Scores. medRxiv, 2021.02.24.21252351 (2021).

87. Zeng, P., Shao, Z. \& Zhou, X. Statistical methods for mediation analysis in the era of high-throughput genomics: Current successes and future challenges. Comput Struct Biotechnol J 19, 3209-3224 (2021).

88. Guo, Y. et al. Genetically predicted body mass index and breast cancer risk: Mendelian randomization analyses of data from 145,000 women of European descent. PLoS medicine 13, e1002105 (2016).

89. Pierce, B.L., Kraft, P. \& Zhang, C. Mendelian randomization studies of cancer risk: a literature review. Current epidemiology reports 5, 184-196 (2018).

90. Shen, X. et al. A phenome-wide association and Mendelian Randomisation study of polygenic risk for depression in UK Biobank. Nat Commun 11, 2301 (2020).

91. Richardson, T.G., Harrison, S., Hemani, G. \& Davey Smith, G. An atlas of polygenic risk score associations to highlight putative causal relationships across the human phenome. Elife 8, e43657 (2019). 
medRxiv preprint doi: https://doi.org/10.1101/2022.01.13.22269176; this version posted January 14 , 2022. The copyright holder for this preprint (which was not certified by peer review) is the author/funder, who has granted medRxiv a license to display the preprint in perpetuity. It is made available under a CC-BY-NC-ND 4.0 International license .

1045 92. Beesley, L.J. et al. The emerging landscape of health research based on biobanks linked to electronic health records: Existing resources, statistical challenges, and potential opportunities. Stat Med 39, 773-800 (2020).

1049

93. Wang, C. et al. Ancestry estimation and control of population stratification for sequence-based association studies. Nat Genet 46, 409-15 (2014).

94. Li, J.Z. et al. Worldwide human relationships inferred from genome-wide patterns of variation. Science 319, 1100-4 (2008).

95. Manichaikul, A. et al. Robust relationship inference in genome-wide association studies. Bioinformatics 26, 2867-73 (2010).

96. Abraham, K.J. \& Diaz, C. Identifying large sets of unrelated individuals and unrelated markers. Source Code Biol Med 9, 6 (2014).

97. McCarthy, S. et al. A reference panel of 64,976 haplotypes for genotype imputation. Nat Genet 48, 1279-83 (2016).

98. Fritsche, L.G. et al. Association of Polygenic Risk Scores for Multiple Cancers in a Phenome-wide Study: Results from The Michigan Genomics Initiative. Am J Hum Genet 102, 1048-1061 (2018).

99. Carroll, R.J., Bastarache, L. \& Denny, J.C. R PheWAS: data analysis and plotting tools for phenome-wide association studies in the R environment. Bioinformatics 30, 2375-6 (2014).

100. Ho, D.E., Imai, K., King, G. \& Stuart, E.A. Matchlt: Nonparametric Preprocessing for Parametric Causal Inference. Journal of Statistical Software 42, 1-28 (2011).

101. Sudlow, C. et al. UK biobank: an open access resource for identifying the causes of a wide range of complex diseases of middle and old age. PLoS Med 12, e1001779 (2015).

102. Bycroft, C. et al. Genome-wide genetic data on $~ 500,000$ UK Biobank participants. bioRxiv (2017).

103. Zhou, W. et al. Efficiently controlling for case-control imbalance and sample relatedness in large-scale genetic association studies. Nat Genet 50, 1335-1341 (2018).

104. Michailidou, K. et al. Association analysis identifies 65 new breast cancer risk loci. Nature 551, 92-94 (2017).

105. Levey, A.S. et al. A new equation to estimate glomerular filtration rate. Ann Intern Med 150, 604-12 (2009).

106. Buniello, A. et al. The NHGRI-EBI GWAS Catalog of published genome-wide association studies, targeted arrays and summary statistics 2019. Nucleic acids research 47, D1005-D1012 (2019).

107. Zhou, X. A unified framework for variance component estimation with summary statistics in genome-wide association studies. The annals of applied statistics 11, 2027 (2017).

108. Zhou, X. \& Stephens, M. Genome-wide efficient mixed-model analysis for association studies. Nature genetics 44, 821-824 (2012).

109. Rao, C.R. Estimation of variance and covariance components-MINQUE theory. Journal of multivariate analysis 1, 257-275 (1971).

110. Rao, C.R. Estimation of heteroscedastic variances in linear models. Journal of the American Statistical Association 65, 161-172 (1970). 
1090 111. Lee, S.H., Wray, N.R., Goddard, M.E. \& Visscher, P.M. Estimating missing heritability for disease from genome-wide association studies. Am J Hum Genet 88, 294-305 (2011).

112. Nagelkerke, N.J. A note on a general definition of the coefficient of determination. Biometrika 78, 691-692 (1991).

113. Fritsche, L.G. et al. Cancer PRSweb: an online repository with Polygenic Risk Scores for major cancer traits and their evaluation in two independent biobanks. The American Journal of Human Genetics 107, 815-836 (2020).

114. Lambert, S.A. et al. The Polygenic Score Catalog as an open database for reproducibility and systematic evaluation. Nature Genetics 53, 420-425 (2021).

115. Little, J. et al. STrengthening the REporting of Genetic Association Studies (STREGA): an extension of the STROBE statement. PLoS Med 6, e22 (2009). 


\section{Web Resources}

1105 The Michigan Genomics Initiative (MGI), https://precisionhealth.umich.edu/our-

1106 research/michigangenomics/

1107 CMS Chronic Condition Warehouse, https://www2.ccwdata.org/web/guest/home/

1108 UCSC Genome Browser Store, https://genome-store.ucsc.edu

1109 Locuszoom, https://github.com/statgen/locuszoom

1110 Rprs, https://github.com/statgen/Rprs

1111 ExPRSweb, https://exprsweb.sph.umich.edu

1112 NHGRI-EBI GWAS Catalog, https://www.ebi.ac.uk/gwas/summary-statistics

1113 FinnGen consortium, https://www.finngen.fi/en/access_results

1114 UKB GWAS (Lee Lab): https://www.leelabsg.org/resources

1115 UKB GWAS (Neale Lab): https://github.com/Nealelab/UK_Biobank_GWAS

1116 Gemma and DBSLMM: https://zlab.org/software.html

1117 lassosum: https://github.com/tshmak/lassosum

1118 PRS-CS: https://github.com/getian107/PRScs

1119 PLINK: https://www.cog-genomics.org/plink2 


\section{Figure Legends}

1122 Figure 1. Flow chart of ExPRS construction, evaluation, and selection.

1123 Figure 2. Prediction performance of the five applied PRS methods in MGI across

1124 continuous (left) and binary (right) traits. Here, the heatmap shows the relative

1125 prediction performance for each method across traits (values were scaled to $0-1$ range)

1126 for better comparison. Specifically, the prediction performance is quantified using $R^{2}$ for

1127 continuous traits and covariate-adjusted AUC for binary traits. For a fair comparison we

1128 selected the same summary statistic for each method (GWAS with the highest

1129 heritability estimate).

1130 Figure 3. Comparison of the pairwise correlation of 15 ExPRS and their

1131 corresponding continuous traits in MGI. Heatmap displays the pairwise correlation

1132 between (A)15 continuous exposures in MGI; (B) ExPRSs; and (C) Exposures (y-axis)

1133 and ExPRSs (x-axis). Here, Pairwise Spearman correlation with nominally significant

1134 association $\mathrm{P}$ values $(<=0.05)$ are shown. Fasting plasma glucose (exposure and

1135 ExPRS) was excluded due to the exposures low sample size in MGI.

1136 Figure 4. ExPRS PheWAS and Exclusion ExPRS PheWAS as an example for

1137 continuous traits in MGI. (A) ExPRS PheWAS plot for BMI ExPRS (B) Exclusion

1138 ExPRS PheWAS plot is shown for using BMI ExPRS as predictor among the individuals

1139 with normal BMI value $(18.5 \sim 24.9 \mathrm{~kg} / \mathrm{m} 2)$ (C) Trait PheWAS plot is shown for BMI trait

1140 (D) Exclusion Trait PheWAS plot is shown for using BMI trait as predictor among the

1141 individuals with normal BMI value. The axis breaks were chosen so that the 10 
1142 strongest signals fall in the top scale ( $y$-axis breaks for the four panels at - $\log _{10} \mathrm{P}$ are 84 ,

114313,540 , and 12, respectively).

1144 Figure 5. Comparisons of the prediction performance of different predictors for

1145 common chronic conditions in MGI cohort. AAUC paired with 95\% confidence

1146 interval for condition specific PRS (YPRS, red), combined ExPRSs (multiExPRS, blue)

1147 and YPRS + multiExPRS (orange) were shown as forest plot. Each bar represents the

$114895 \%$ interval for the AAUC with the dot represents the AAUC estimate. 


\section{Tables.}

1150 Table 1. Demographics and Clinical Characteristics of the Analytic Datasets

1151

\begin{tabular}{lll}
\hline Characteristic & MGI & UKB
\end{tabular}

\section{Demographics}

Study Type

$\mathrm{n}$

Females, n (\%)

Mean age, years (SD)

Neighborhood Deprivation Index (SD)

Townsend Deprivation Index (TDI)

\section{Visits / Measurements}

Median number of visits per participant

Median time (years) between first and last visit

Median lab orders per participant

Body Mass Index (BMI)

Underweight $(\mathrm{BMI}<18.5)$, $\mathrm{n}(\%)$

Normal (BMI 18.5-24.9), n (\%)

Overweight (BMI > 25.0), n (\%)

\section{Smoking Status}

Yes

No

\section{Selected common chronic conditions}

Hypertension, n (\%)

Diabetes

Lung Cancer
Hospital-based

46,782

$24,454(52.3 \%)$

56.7 (16.4)

$0.9(0.6)$

not available

45

5.5

59

$29.9(7.1)$

$498(1.1 \%)$

11,349 (24.3\%)

$34,916(74.7 \%)$

$22919(49.2 \%)$

$23,744(50.8 \%)$

$160,954(39.4 \%)$

247,641 (60.6\%)

* Based on all available dates of first in-patient diagnoses 
1154 Table 2. Overview of the 28 Included Exposures Traits. Number of included 1155 discovery GWASs, estimated heritability (liability scale) and sample size of PRS 1156 evaluation cohorts are shown. Details can be found in Tables S1-S3.

\begin{tabular}{|c|c|c|c|c|c|c|c|}
\hline \multirow[t]{2}{*}{ Exposure } & \multirow[t]{2}{*}{ Category } & \multicolumn{2}{|c|}{ Discovery GWAS } & \multicolumn{2}{|c|}{ Heritability* } & \multicolumn{2}{|c|}{$\begin{array}{l}\text { Evaluation Cohort } \\
\text { Sample Sizes } \\
\text { Cases / Controls or } \\
\text { Total }\end{array}$} \\
\hline & & Meta-analysis & UKB & $h_{g}{ }^{2}$ & S.E. & MGI & UKB \\
\hline \multicolumn{8}{|l|}{ Continuous Traits } \\
\hline HDL cholesterol & Cardiovascular & $\cdots$ & 2 & 0.228 & 0.021 & 18639 & $\cdots$ \\
\hline LDL cholesterol & Cardiovascular & $\ldots$ & 2 & 0.113 & 0.016 & 18576 & $\ldots$ \\
\hline Triglycerides & Cardiovascular & $\ldots$ & 2 & 0.200 & 0.022 & 19184 & $\cdots$ \\
\hline Total cholesterol & Cardiovascular & $\cdots$ & 2 & 0.131 & 0.017 & 18231 & $\cdots$ \\
\hline PUFAs & Cardiovascular & 1 & $\ldots$ & 0.148 & 0.081 & $\ldots$ & 174277 \\
\hline CRP & Cardiovascular & 1 & 2 & 0.198 & 0.106 & 10292 & 389826 \\
\hline eGFR & Renal Biomarker & 1 & & 0.051 & 0.004 & 43039 & 390449 \\
\hline Creatinine & Renal Biomarker & 3 & 4 & 0.260 & 0.038 & 40792 & 390449 \\
\hline Cystatin C & Renal Biomarker & 3 & 2 & 0.230 & 0.025 & 213 & 390609 \\
\hline Vitamin D & Vitamin Levels & 1 & 4 & 0.100 & 0.017 & 13854 & 373768 \\
\hline Vitamin B12 & Vitamin Levels & 1 & 2 & 0.023 & 0.293 & 8626 & 174277 \\
\hline Fasting glucose plasma & Blood Sugar Levels & 2 & $\ldots$ & 0.071 & 0.011 & 570 & $\ldots$ \\
\hline Glucose & Blood Sugar Levels & 2 & 2 & 0.077 & 0.008 & 40801 & 346477 \\
\hline Estradiol & Women's Health & 2 & 2 & 0.033 & 0.007 & 1875 & 61982 \\
\hline Age at menopause & Women's Health & 1 & $\ldots$ & 0.109 & 0.010 & $\ldots$ & 139773 \\
\hline Age at menarche & Women's Health & 1 & $\ldots$ & 0.109 & 0.007 & $\ldots$ & 220885 \\
\hline $\mathrm{BMI}$ & Anthropometric & $\ldots$ & 4 & 0.239 & 0.009 & 46763 & $\ldots$ \\
\hline Height & Anthropometric & 2 & 2 & 0.518 & 0.034 & 46699 & 407750 \\
\hline DBP & Vitals & $\cdots$ & 4 & 0.140 & 0.008 & 46148 & $\cdots$ \\
\hline SBP & Vitals & $\ldots$ & 4 & 0.148 & 0.008 & 46144 & $\ldots$ \\
\hline Alcohol Amount & Health Behavior & 1 & 1 & 0.055 & 0.006 & 26666 & 121424 \\
\hline \multicolumn{8}{|l|}{ Binary Traits } \\
\hline Thinness & Anthropometric & 1 & $\ldots$ & 0.133 & 0.034 & $\begin{array}{l}753 / \\
41938\end{array}$ & $\begin{array}{l}3547 / \\
396201\end{array}$ \\
\hline Drinker & Health Behavior & $\cdots$ & 1 & 0.100 & 0.006 & $\begin{array}{l}30900 / / \\
13952\end{array}$ & $\ldots$ \\
\hline Smoker & Health Behavior & 1 & 3 & 0.156 & 0.007 & $\begin{array}{l}22919 / \\
23744\end{array}$ & $\begin{array}{l}246067 \\
\text { / } \\
160791\end{array}$ \\
\hline Type 2 diabetes & Preexisting condition & 3 & 1 & 0.307 & 0.027 & $\begin{array}{l}9843 / \\
32794\end{array}$ & $\begin{array}{l}19780 / \\
386988\end{array}$ \\
\hline Hypertension & Preexisting condition & 2 & 3 & 0.413 & 0.165 & $\begin{array}{l}23158 / \\
23465\end{array}$ & $\begin{array}{l}77740 / \\
329912\end{array}$ \\
\hline Insomnia & Preexisting condition & $\cdots$ & 2 & $\mathrm{n} / \mathrm{a}^{\star *}$ & $\mathrm{n} / \mathrm{a}$ & $\begin{array}{l}5524 / \\
31654\end{array}$ & $\cdots$ \\
\hline Apnoea & Preexisting condition & 1 & 1 & 0.284 & 0.057 & $\begin{array}{l}10909 / \\
31654\end{array}$ & $\begin{array}{l}4460 / \\
403370\end{array}$ \\
\hline
\end{tabular}
CRP: C-reactive protein; eGFR: estimated glomerular filtration rate; BMI: Body mass index; DBP: diastolic blood pressure; SBP: Systolic blood pressure; .... not available; 
1164 Table 3. Top ranked ExPRS in MGI. Details about the underlying discovery GWAS 1165 study, can be found in Table S2.

\begin{tabular}{|c|c|c|c|c|c|c|c|}
\hline Exposure & $\begin{array}{l}\text { Discovery } \\
\text { GWAS }\end{array}$ & Method & $\begin{array}{l}\text { \# SNPs } \\
\text { in } \\
\text { ExPRS }\end{array}$ & $\begin{array}{l}\text { Association } \\
\mathbf{P}\end{array}$ & $\begin{array}{l}\text { Brier } \\
\text { Score }\end{array}$ & $\begin{array}{l}\text { Pearson's } \\
\text { r }\end{array}$ & $\begin{array}{c}\text { Adjusted AUC } \\
(95 \% \mathrm{Cl})\end{array}$ \\
\hline \multicolumn{8}{|l|}{$\begin{array}{l}\text { Continuous } \\
\text { Traits }\end{array}$} \\
\hline HDL & UK Biobank & PRS-CS & 1113830 & 1.3E-294 & $\ldots$ & 0.311 & $\ldots$ \\
\hline LDL & UK Biobank & DBSLMM & 8918470 & $4.1 \mathrm{E}-174$ & $\ldots$ & 0.274 & $\ldots$ \\
\hline $\mathrm{TG}$ & UK Biobank & DBSLMM & 8924773 & 6.9E-304 & $\ldots$ & 0.348 & $\ldots$ \\
\hline $\mathrm{TC}$ & UK Biobank & PRS-CS & 1113831 & $3.2 E-168$ & $\ldots$ & 0.265 & $\ldots$ \\
\hline CRP & UK Biobank & PRS-CS & 1113831 & $6.2 \mathrm{E}-30$ & $\ldots$ & 0.155 & $\ldots$ \\
\hline eGFR & Meta-analysis & PRS-CS & 1113831 & $3.8 \mathrm{E}-150$ & $\ldots$ & 0.13 & $\ldots$ \\
\hline Creatinine & UK Biobank & PRS-CS & 1113831 & 4.6E-189 & $\ldots$ & 0.174 & $\ldots$ \\
\hline Vitamin D & UK Biobank & $\mathrm{C}+\mathrm{T}(\mathrm{GT})$ & 500 & $9.0 \mathrm{E}-46$ & $\ldots$ & 0.166 & $\ldots$ \\
\hline Vitamin B12 & UK Biobank & $\mathrm{C}+\mathrm{T}(\mathrm{DS})$ & 9 & 0.0011 & $\ldots$ & 0.049 & $\ldots$ \\
\hline FPG & Meta-analysis & $\mathrm{C}+\mathrm{T}(\mathrm{GT})$ & 273 & 0.0095 & $\ldots$ & 0.099 & $\ldots$ \\
\hline Glucose & UK Biobank & PRS-CS & 1113830 & $8.4 \mathrm{E}-112$ & $\ldots$ & 0.156 & $\ldots$ \\
\hline Estradiol & UK Biobank & PRS-CS & 1113823 & 0.046 & $\ldots$ & 0.0664 & $\ldots$ \\
\hline BMI & UK Biobank & PRS-CS & 1113832 & 5.3E-607 & $\ldots$ & 0.319 & $\ldots$ \\
\hline Height & UK Biobank & PRS-CS & 1113832 & $2.2 E-1988$ & $\ldots$ & 0.373 & $\ldots$ \\
\hline DBP & UK Biobank & PRS-CS & 1113831 & $4.8 \mathrm{E}-170$ & $\ldots$ & 0.166 & $\ldots$ \\
\hline SBP & UK Biobank & PRS-CS & 1113831 & 1.7E-189 & $\ldots$ & 0.172 & $\ldots$ \\
\hline Alcohol Amt. & Meta-analysis & PRS-CS & 1116497 & $1.4 \mathrm{E}-18$ & $\ldots$ & 0.0746 & $\ldots$ \\
\hline \multicolumn{8}{|l|}{ Binary Traits } \\
\hline Thinness & Meta-analysis & $\mathrm{C}+\mathrm{T}(\mathrm{DS})$ & 256 & 0.044 & 0.018 & $\cdots$ & $\begin{array}{c}0.532 \\
(0.503,0.561)\end{array}$ \\
\hline Drinker & UK Biobank & PRS-CS & 1113832 & $5.1 \mathrm{E}-29$ & 0.212 & $\ldots$ & $\begin{array}{c}0.547 \\
(0.539,0.536)\end{array}$ \\
\hline Smoker & Meta-analysis & PRS-CS & 1109786 & $1.10 \mathrm{E}-170$ & 0.232 & $\ldots$ & $\begin{array}{c}0.605 \\
(0.598,0.612)\end{array}$ \\
\hline $\mathrm{T} 2 \mathrm{D}$ & Meta-analysis & PRS-CS & 945820 & $1.10 \mathrm{E}-159$ & 0.139 & $\ldots$ & $\begin{array}{c}0.637 \\
(0.621,0.653)\end{array}$ \\
\hline Hypertension & UK Biobank & PRS-CS & 1113832 & $6.4 \mathrm{E}-213$ & 0.182 & $\cdots$ & $\begin{array}{c}0.630 \\
(0.622,0.639)\end{array}$ \\
\hline Insomnia & UK Biobank & PRS-CS & 1065129 & 5.0E-06 & 0.126 & $\ldots$ & $\begin{array}{c}0.524 \\
(0.513,0.536)\end{array}$ \\
\hline Sleep apnea & UK Biobank & PRS-CS & 1111194 & $5.8 \mathrm{E}-10$ & 0.182 & $\ldots$ & $\begin{array}{c}0.527 \\
(0.517,0.536)\end{array}$ \\
\hline
\end{tabular}




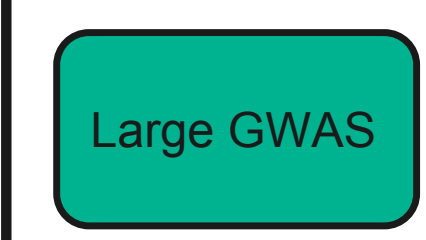

UK Biobank GWAS

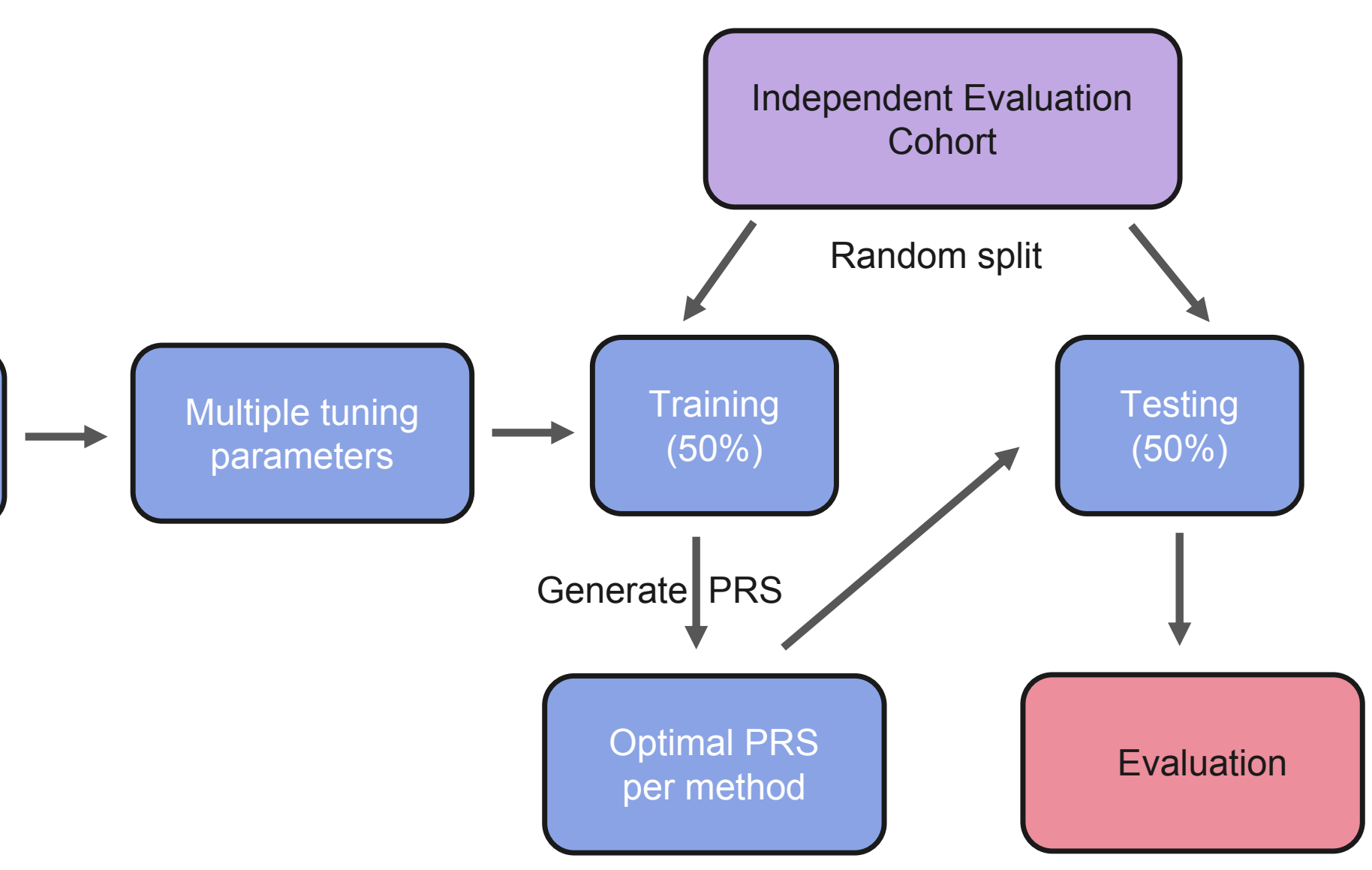

Adjust summary statistics with PRS methods

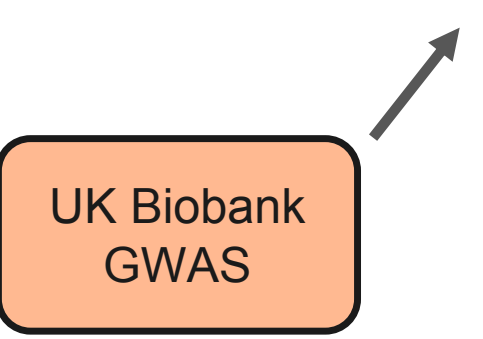


Figure 2

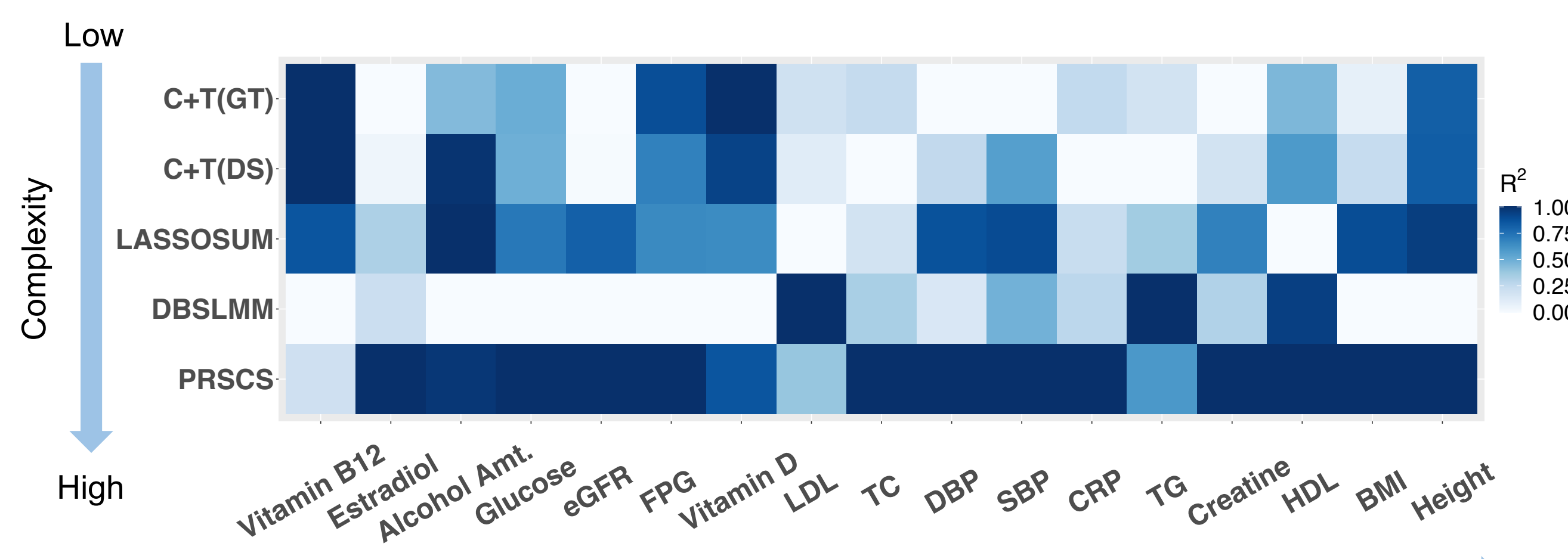


(A) Exposure v. Exposure
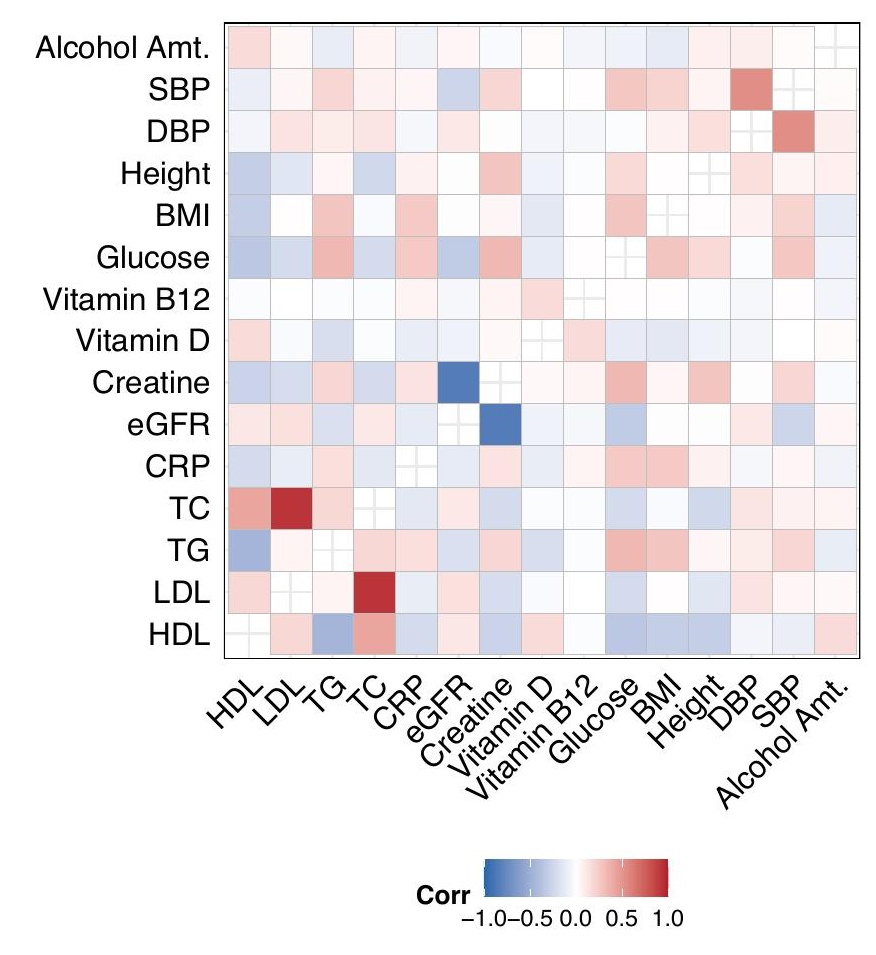

(B)

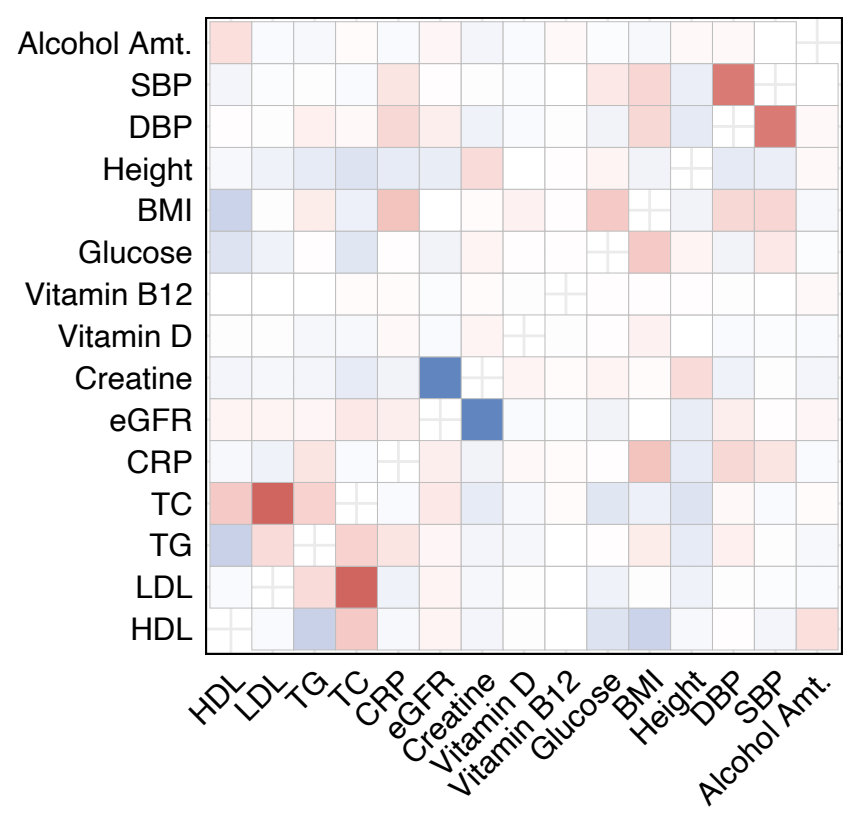

(C)

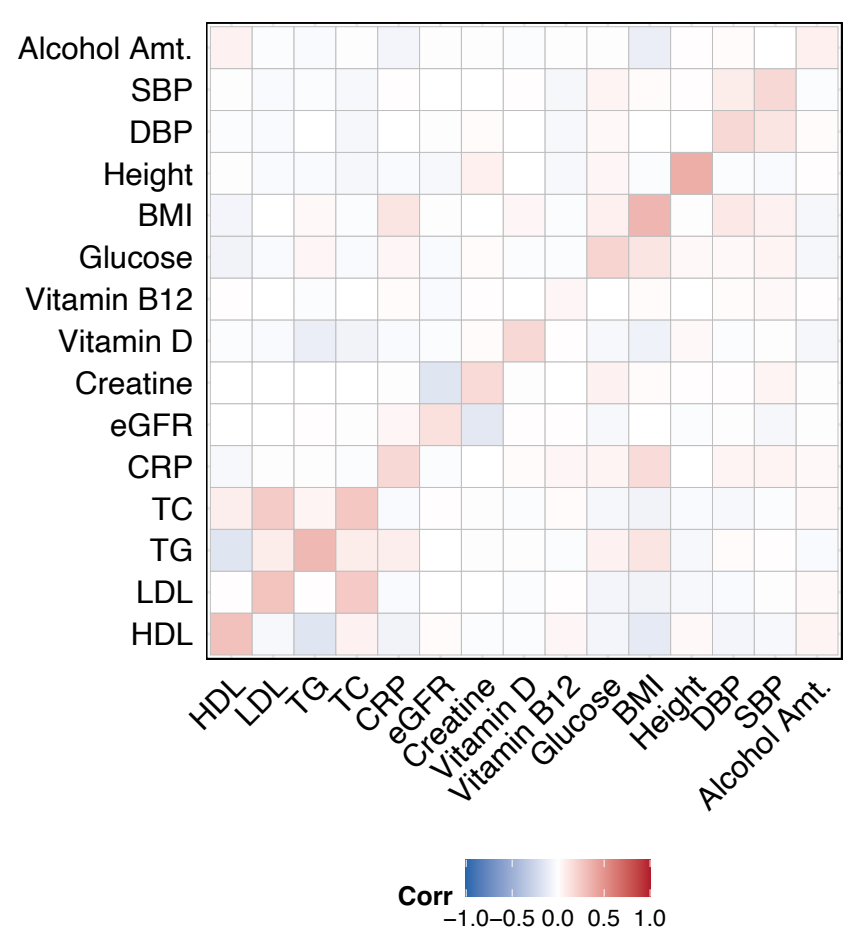

ExPRS v. Exposure

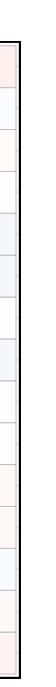

(1)

. 


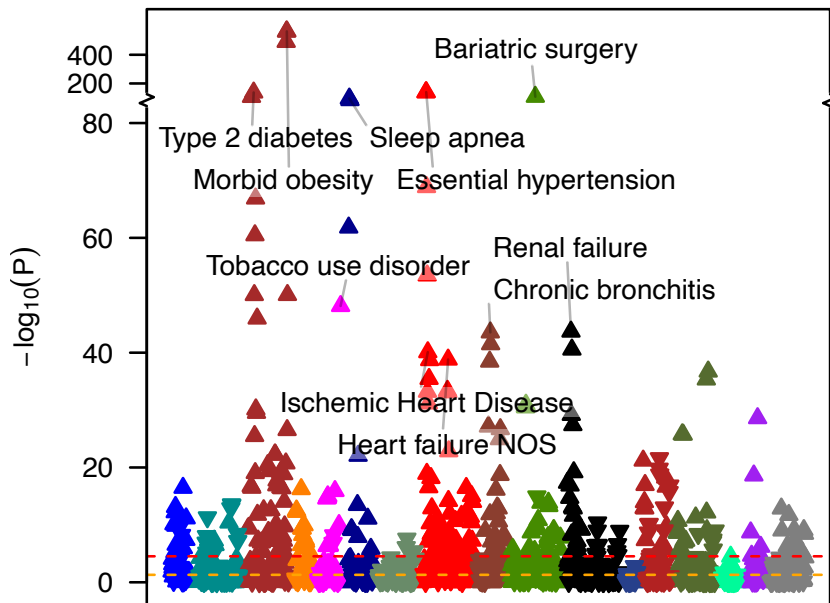

C

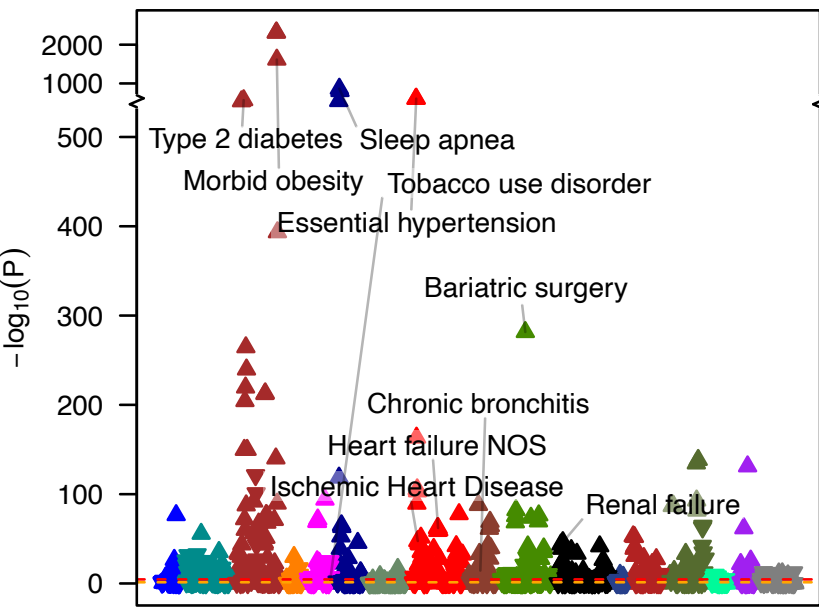

B

BMI PRS / Normal Range BMI Only

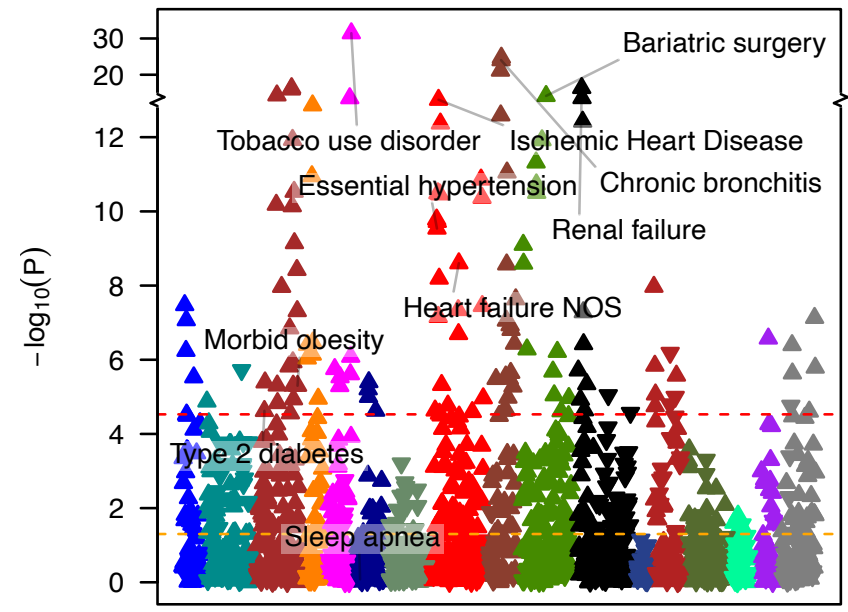

D

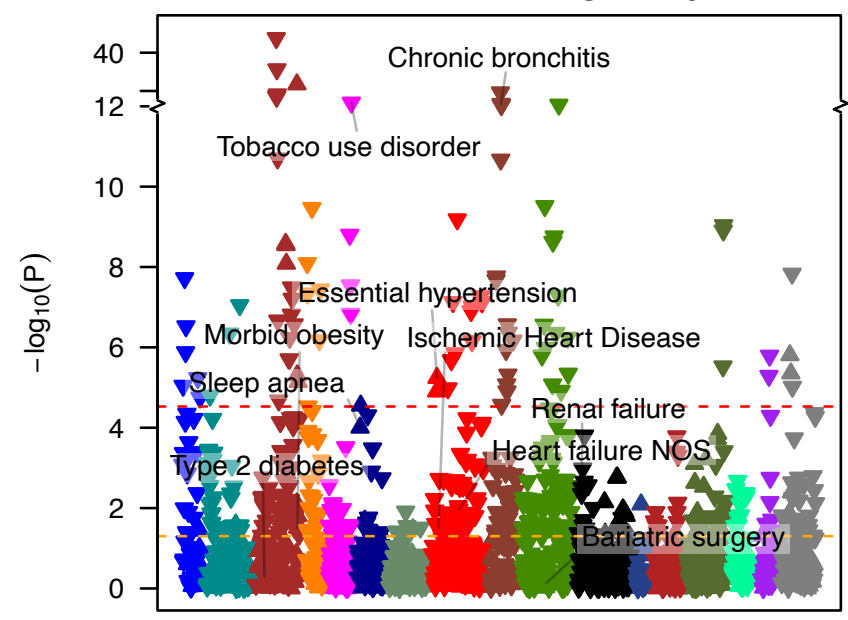

PheCode Categories / Legend

- Infectious Diseases

- Neoplasms

- Endocrine/Metabolic

- Hematopoietic

- Mental Disorders
- Neurological

- Sense Organs

- Circulatory System

- Respiratory

- Digestive
- Genitourinary

- Pregnancy Complications - Injuries \& Poisonings

- Dermatologic

- Musculoskeletal

- Congenital Anomalies $\triangle$ Positively associated $\nabla$ Negatively associated 
Stroke/Transient Ischemic Attack

Heart Failure

Glaucoma

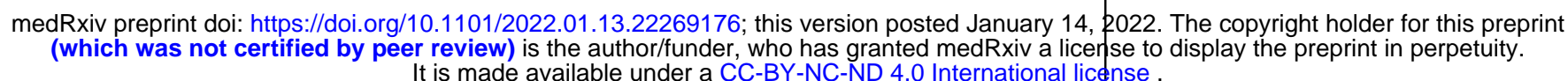

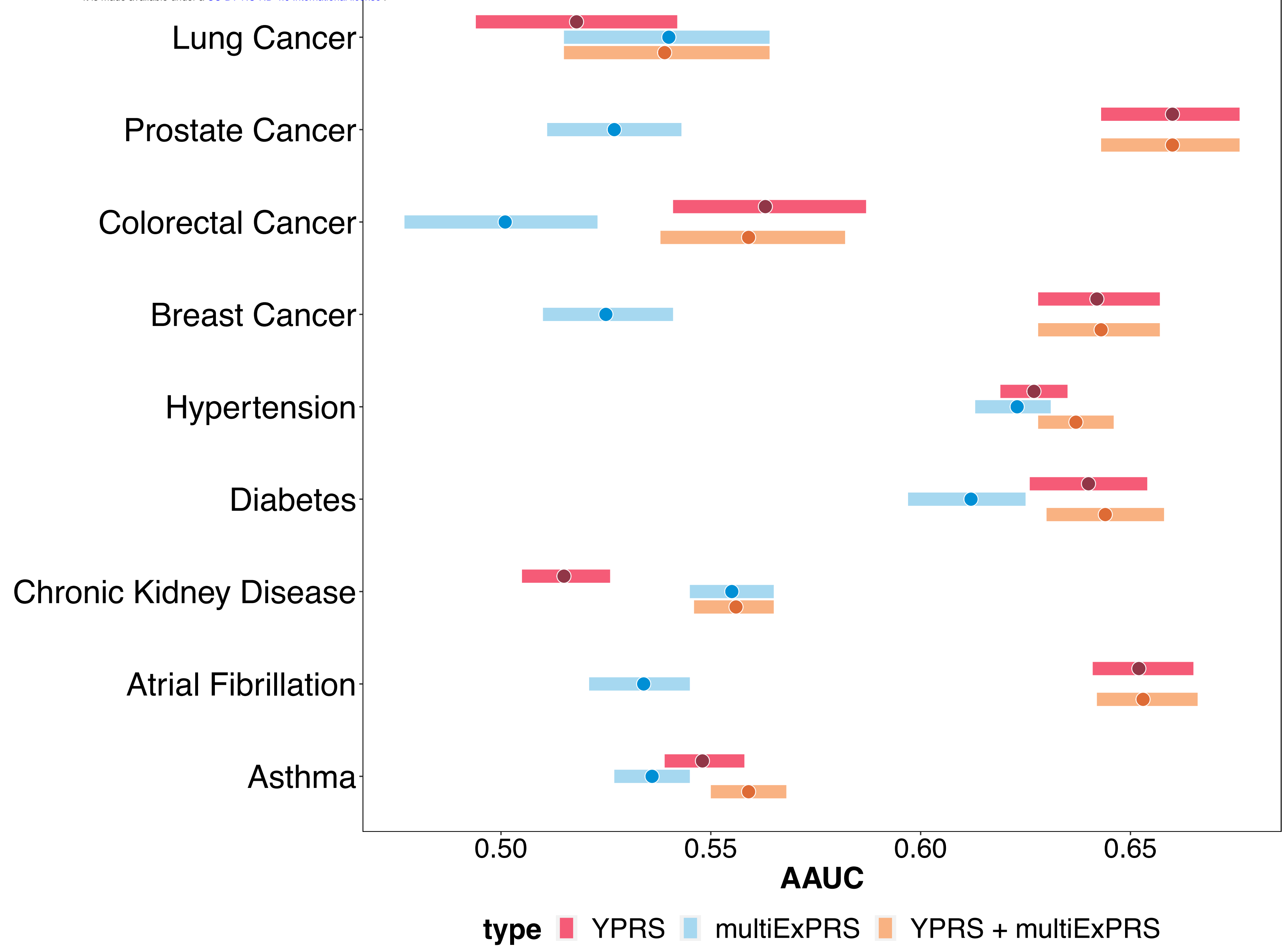

